\title{
FUNCTIONAL EQUATION FOR THE SELMER GROUP OF NEARLY ORDINARY HIDA DEFORMATION OF HILBERT MODULAR FORMS.
}

\author{
SOMNATH JHA, DIPRAMIT MAJUMDAR
}

\begin{abstract}
We establish a duality result proving the 'functional equation' of the characteristic ideal of the Selmer group associated to a nearly ordinary Hilbert modular form over the cyclotomic $\mathbb{Z}_{p}$ extension of a totally real number field. Further, we use this result to establish a duality or algebraic 'functional equation' for the 'big' Selmer groups associated to the corresponding nearly ordinary Hida deformation. The multivariable cyclotomic Iwasawa main conjecture for nearly ordinary Hida family of Hilbert modular forms is not established yet and this can be thought of as an evidence to the validity of this Iwasawa main conjecture. We also prove a functional equation for the 'big' Selmer group associated to an ordinary Hida family of elliptic modular forms over the $\mathbb{Z}_{p}^{2}$ extension of an imaginary quadratic field.
\end{abstract}

\section{Contents}

Introduction 1

1. Preliminaries 5

1.1. Preliminary results on Hilbert Modular Forms 5

$\begin{array}{ll}\text { 1.2. Various Selmer groups } & 10\end{array}$

2. functional equation for a Hilbert modular form 13

3. functional equation for a nearly ordinary Hida family 17

4. Results over $\mathbb{Z}_{p}^{2}$ extension $\quad 25$

References $\quad 31$

\section{INTRODUCTION}

We fix an odd rational prime $p$ and $N$ a natural number prime to $p$. Throughout, we fix an embedding $\iota_{\infty}$ of a fixed algebraic closure $\overline{\mathbb{Q}}$ of $\mathbb{Q}$ into $\mathbb{C}$ and also an embedding $\iota_{l}$ of $\overline{\mathbb{Q}}$ into a fixed algebraic closure $\overline{\mathbb{Q}}_{\ell}$ of the field $\mathbb{Q}_{\ell}$ of the $\ell$-adic numbers, for every prime $\ell$. Let $F$ denote a totally real number field and $K$ denote an imaginary quadratic field. For any number field $L, S_{L}$ will denote a finite set of places of $L$ containing the primes dividing $N p$. The cyclotomic $\mathbb{Z}_{p}$ extension of $L$ will be denoted by $L_{\text {cyc }}$ and the unique $\mathbb{Z}_{p}^{2}$ extension of $K$ will be denoted by $K_{\infty}$. Set $\Gamma:=\operatorname{Gal}\left(L_{\text {cyc }} / L\right) \cong \mathbb{Z}_{p}$ and $\Gamma_{K}:=\operatorname{Gal}\left(K_{\infty} / K\right) \cong \mathbb{Z}_{p}^{2}$. Let $B$ be a commutative, complete, noetherian, normal, local ring of characteristic 0 with finite residue field of characteristic $p$. We will denote by $B[[\Gamma]]$ (resp. $B\left[\left[\Gamma_{K}\right]\right]$ ) the Iwasawa 
algebra of $\Gamma$ (resp. $\Gamma_{K}$ ) with coefficient in $B$. Let $M$ be a finitely generated torsion $B[[\Gamma]]$ (resp. $B\left[\left[\Gamma_{K}\right]\right]$ ) module. Then $M^{\iota}$ denote the $B[[\Gamma]]$ (resp. $B\left[\left[\Gamma_{K}\right]\right]$ ) module whose underlying abelian group is the same as $M$ but the $\Gamma$ (resp. $\Gamma_{K}$ ) action is changed via the involution sending $\gamma \mapsto \gamma^{-1}$ for every $\gamma \in \Gamma$ (resp. $\gamma \in \Gamma_{K}$ ). We denote the characteristic ideal of $M$ in $B[[\Gamma]]$ (resp. $\left.B\left[\left[\Gamma_{K}\right]\right]\right)$ ) by $C h_{B[[\Gamma]]}(M)$ (resp. $\left.C h_{B\left[\left[\Gamma_{K}\right]\right]}(M)\right)$. The main results of the article is the following theorem.

Theorem (Theorem 3.10 and Theorem 4.9). Let $\mathcal{R}$ be a branch of Hida's universal nearly ordinary Hecke algebra associated to nearly ordinary Hilbert modular forms (resp. Hida's universal ordinary Hecke algebra associated to ordinary elliptic modular forms). Let $\mathcal{T}_{\mathcal{R}}$ be a $G_{F}$ (resp. $G_{\mathbb{Q}}$ ) invariant lattice associated to 'big' Galois representation $\left(\rho_{\mathcal{R}}, \mathcal{V}_{\mathcal{R}}\right)$ and set $\mathcal{T}_{\mathcal{R}}^{*}:=\operatorname{Hom}_{\mathcal{R}}\left(\mathcal{T}_{\mathcal{R}}, \mathcal{R}(1)\right.$ ). Let $\mathcal{X}\left(\mathcal{T}_{\mathcal{R}} / F_{\text {cyc }}\right)$ (resp. $\mathcal{X}\left(\mathcal{T}_{\mathcal{R}} / K_{\infty}\right)$ ) denote the dual Selmer group of $\mathcal{R}$ over $F_{\text {cyc }}$ (resp. $\left.K_{\infty}\right)$. Then under certain conditions, as ideals in $\mathcal{R}[[\Gamma]]$ (resp. $\mathcal{R}\left[\left[\Gamma_{K}\right]\right]$ ), we have the equality

$$
C h_{\mathcal{R}[[\Gamma]]}\left(\mathcal{X}\left(\mathcal{T}_{\mathcal{R}} / F_{\text {cyc }}\right)\right)=C h_{\mathcal{R}[[\Gamma]]}\left(\mathcal{X}\left(\mathcal{T}_{\mathcal{R}}^{*} / F_{\text {cyc }}\right)^{\iota}\right)
$$

(resp.

$$
\left.C h_{\mathcal{R}\left[\left[\Gamma_{K}\right]\right]}\left(\mathcal{X}\left(\mathcal{T}_{\mathcal{R}} / K_{\infty}\right)\right)=C h_{\mathcal{R}\left[\left[\Gamma_{K}\right]\right]}\left(\mathcal{X}\left(\mathcal{T}_{\mathcal{R}}^{*} / K_{\infty}\right)^{\iota}\right) .\right)
$$

Theorem 3.10 and Theorem 4.9 generalizes [J-P, Theorem 5.2]. An ingredient in the proof of Theorem 3.10 is Theorem 2.10, where we prove a functional equation for the Selmer group of a single Hilbert modular form. Theorem 2.10 is a variant of [Gr1, Theorem 2] and [Pe, Theorem 4.2.1]. The motivation for the above theorems is explained below.

Let $E$ be an elliptic curve defined over $\mathbb{Q}$ with good ordinary reduction at an odd prime $p$. Let $L_{E}$ be the complex $L$ function of $E$. Then by modularity theorem, $E$ is modular and $L_{E}$ coincides with the $L$-function of a weight 2 newform of level $N_{E}$, where $N_{E}$ is the conductor of $E$. Moreover, $L_{E}$ has analytic continuation to all of $\mathbb{C}$ and if we set

$$
\Lambda_{E}(s):=N_{E}^{s / 2}(2 \pi)^{-s} \Gamma(s) L_{E}(s)
$$

to be the completed $L$-function of $E$, where $\Gamma(s)$ is the usual $\Gamma$ function, then (due to Hecke)

$$
\Lambda_{E}(2-s)= \pm \Lambda_{E}(s), s \in \mathbb{C} .
$$

Thus $L_{E}$ satisfies a functional equation connecting values at $s$ and $2-s$, where $s \in \mathbb{C}$. Let $\phi$ vary over the Dirichlet characters of $\Gamma=\operatorname{Gal}\left(\mathbb{Q}_{\text {cyc }} / \mathbb{Q}\right)$ i.e. $\phi \in \hat{\Gamma}$, then the twisted $L$-function $L_{E}(s, \phi)$, will also satisfy a functional equation similar to (1) connecting the values of $L_{E}(2-s, \phi)$ and $L_{E}\left(s, \phi^{-1}\right)$. In particular, $L_{E}(1, \phi)$ and $L_{E}\left(1, \phi^{-1}\right)$ are related by a functional equation.

Now, by the work of Mazur and Swinnerton-Dyer [M-SD], the $p$-adic $L$-function of $E$ exists. Let $g_{E}(T)$ be the power series representation of the $p$-adic $L$-function of $E$ in $\mathbb{Z}_{p}[[\Gamma]] \otimes \mathbb{Q}_{p} \cong \mathbb{Z}_{p}[[T]] \otimes \mathbb{Q}_{p}$. Then we have,

- $g_{E}(0)=\left(1-\alpha_{p}^{-1}\right)^{2} L_{E}(1) / \Omega_{E}$,

- $g_{E}(\phi(T)-1)=\frac{L_{E}(1, \phi) \beta_{p}{ }^{n}}{\tau(\phi) \Omega_{E}}$, for a character $\phi$ of $\Gamma$ of order $p^{n} \geq 1$. Here $\alpha_{p}+\beta_{p}=a_{p}, \alpha_{p} \beta_{p}=p$ with $p \nmid \alpha_{p}, \Omega_{E}$ is the real period of $E$ and $\tau(\phi)$ is the Gauss sum of $\phi$. 
We assume that $g_{E} \in \mathbb{Z}_{p}[[\Gamma]]$. Then using the above interpolation properties, we can deduce from (1), a functional equation for the $p$-adic $L$-function (also proven in [M-T-T]), given by

$$
g_{E}(T)=u_{E} g_{E}\left(\frac{1}{1+T}-1\right)
$$

where $u_{E}$ is a unit in the ring $\mathbb{Z}_{p}[[\Gamma]] \cong \mathbb{Z}_{p}[[T]]$. In other words, we have an equality of ideals in $\mathbb{Z}_{p}[[\Gamma]]$,

$$
\left(g_{E}(T)\right)=\left(g_{E}\left(\frac{1}{1+T}-1\right)\right) .
$$

By the cyclotomic Iwasawa main conjecture for $E$ (cf. [S-U, $\S 3.5]$ ), the $p$-adic $L$ function $g_{E}(T)$ is a characteristic ideal of the (dual) $p^{\infty}$-Selmer group $X\left(T_{p} E / \mathbb{Q}_{\text {cyc }}\right)$. Thus, we should get

$$
C h_{\mathbb{Z}_{p}[[\Gamma]]}\left(X\left(T_{p} E / \mathbb{Q}_{c y c}\right)\right)=C h_{\mathbb{Z}_{p}[[\Gamma]]}\left(X\left(T_{p} E^{*} / \mathbb{Q}_{c y c}\right)^{\iota}\right),
$$

as ideals in the Iwasawa algebra $\mathbb{Z}_{p}[[\Gamma]]$. (Notice that by Weil pairing, $T_{p} E^{*} \cong T_{p} E$ ).

Indeed, under certain assumptions, (4) is a corollary of the main conjecture of Iwasawa theory for elliptic curves (proven in [S-U, Corollary 3.34], also see [Ka]) together with the fact that the $p$-adic $L$-function $g_{E}$ satisfies functional equation (3). However, for any elliptic curve $E$ defined over $\mathbb{Q}$ which is ordinary at $p,(4)$ was already proven by [Gr1, Theorem 2] (and also independently in [Pe, Theorem 4.2.1]) purely algebraically and without assuming the existence of $g_{E}$. The proofs uses duality and pairing in cohomology, (like the Poitou-Tate duality, generalized Cassels-Tate pairing of Flach) among other tools.

Now for any compatible system of $l$-adic representations associated to a motive, a complex $L$-function is defined and one can think of similar questions. For example, for a normalized cuspidal Hilbert eigenform $f$, which is nearly ordinary at primes $\mathfrak{p}$ of a totally real number field $F$ dividing the prime $p$, one can associate a compatible system of $l$-adic representation. Furthermore, one can define a Selmer group $X\left(T_{f} / F_{\text {cyc }}\right)$ using the Galois representation of the Galois group $G_{F}:=\operatorname{Gal}(\bar{F} / F)$. Under suitable conditions (for example, non-critical slope), the $p$-adic $L$-function for $f$, which interpolates the complex $L$-function, exists (see [Di], also see [Sa]); and a precise Iwasawa main conjecture for $f$ over $F_{\text {cyc }}$ can also be formulated (for example, see [Wa]). However, this cyclotomic Iwasawa main conjecture for $f$ is not proven yet. In Theorem 2.10, we prove the functional equation for the characteristic ideal of the Selmer group of $f$ i.e.

$$
C h_{O_{f}[[\Gamma]]}\left(X\left(T_{f} / F_{\text {cyc }}\right)\right)=C h_{O_{f}[[\Gamma]]}\left(X\left(T_{f}^{*} / F_{\text {cyc }}\right)^{\iota}\right),
$$

algebraically (without assuming the existence of the $p$-adic $L$-function or the Iwasawa main conjecture of $f$ ). Thus, Theorem 2.10 can be thought of as a modest evidence towards the validity for the cyclotomic Iwasawa main conjecture for $f$.

Now, let us consider the nearly ordinary Hida deformation of Hilbert modular forms. A 'several variable' $p$-adic $L$-function (say $\mathcal{L}^{p}$ ) associated to (a branch $\mathcal{R}$ of the) nearly ordinary Hida family $\mathbf{H}_{\mathcal{N}, O}$ over $F_{\text {cyc }}$ will interpolate the special values of the complex $L$-functions of the various individual nearly ordinary normalized 
cuspidal Hilbert eigenforms lying in the family (cf. [Oc4], [Di]). Hence, $\mathcal{L}^{p}$ should also satisfy a functional equation. Again, by the 'several variable' Iwasawa main conjecture over $F_{\text {cyc }}$ for a nearly ordinary Hida family $\mathbf{H}_{\mathcal{N}, O}$ of Hilbert modular forms (cf. [Wa]), $\mathcal{L}_{p}$ should be a characteristic ideal of the 'big' Selmer group $\mathcal{X}\left(\mathcal{T}_{\mathcal{R}} / F_{\text {cyc }}\right)$ of the branch $\mathcal{R}$ of the nearly ordinary Hida family. Thus, we would expect to get a 'functional equation' stating

$$
C h_{R[[\Gamma]]}\left(\mathcal{X}\left(\mathcal{T}_{\mathcal{R}} / F_{\text {cyc }}\right)\right)=C h_{R[[\Gamma]]}\left(\mathcal{X}\left(\mathcal{T}_{\mathcal{R}}^{*} / F_{\text {cyc }}\right)^{\iota}\right)
$$

where $\Gamma=\operatorname{Gal}\left(F_{\text {cyc }} / F\right)$. Again, we will prove this fact algebraically (without any assumption on the analytic side) in Theorem 3.10 and thus, in turn, this can be thought of as a modest evidence for the validity of the Iwasawa main conjecture for the nearly ordinary Hida deformation of Hilbert modular forms over $F_{\text {cyc }}$.

From an purely algebraic point of view of duality and pairing in cohomology, Theorem 3.10 can also be thought as a direct generalization of result of [Gr1, Theorem $2]$ in the nearly ordinary Hida deformation setting.

An entirely parallel argument as above, in the setting of ordinary Hida deformation of elliptic modular forms over the $\mathbb{Z}_{p}^{2}$ extension of an imaginary quadratic field $K$, works as the motivation for Theorem 4.9. (Functional equation for elliptic modular forms over the cyclotomic $\mathbb{Z}_{p}$ extension of $K$ was discussed in [J-P, Theorem 5.2].) In this case though, we would like to stress that for an imaginary quadratic field $K$, under certain hypotheses, the three variable Iwasawa main conjecture over $K_{\infty}$ for an ordinary Hida family of elliptic modular form has been proven in $[\mathrm{S}-\mathrm{U}$, $\S 3.6 .3]$. Indeed, in that article a suitable three variable $p$-adic $L$-function for the ordinary Hida family has been constructed ( $[\mathrm{S}-\mathrm{U}, \S 3.4 .5])$. It is known that this 3 variable $p$-adic $L$-function should satisfy a functional equation. Thus, at least in principle, the work of [S-U] should also establish the equality

$$
C h_{\mathcal{R}\left[\left[\Gamma_{K}\right]\right]}\left(\mathcal{X}\left(T_{\mathcal{R}} / K_{\infty}\right)\right)=C h_{\mathcal{R}\left[\left[\Gamma_{K}\right]\right]}\left(\mathcal{X}\left(T_{\mathcal{R}}^{*} / K_{\infty}\right)^{\iota}\right)
$$

in $\mathcal{R}\left[\left[\Gamma_{K}\right]\right]$. However, we would like to mention that our proof of the functional equation for the Selmer group $\mathcal{X}\left(\mathcal{T}_{\mathcal{R}} / K_{\infty}\right)$ (Theorem 4.9) is simple and we do not need to make use of the vast tools involved in the proof of the 3 variable main conjecture of [S-U]. Moreover, in Theorem 4.9, we do not need some of the hypotheses of the proof of the main conjecture (see $[\mathrm{S}-\mathrm{U}, \S 3.6]$ ).

The key idea of the proof of Theorem 2.10 is to use generalized Cassels-Tate pairing of Flach along with a "control theorem" (Theorem 2.3). The central idea of the proof of Theorem 3.10 (and Theorem 4.9) can be explained in three steps. First, we show that for infinitely many arithmetic points the specialization map is a pseudo-isomorphism. Secondly, we use the fact that functional equation holds at the fibre for infinitely many arithmetic specialization. Finally, we use some suitable lifting techniques, generalization of results of [Oc3], to obtain our results. This gives a simple proof of the desired functional equation of the 'big' Selmer group of the nearly ordinary Hida family.

The structure of the article is as follows. In section 1, we discuss some preliminary results in two parts. In subsection 1.1, we discuss preliminaries related to the Hida deformation for nearly ordinary Hilbert modular forms and ordinary elliptic modular 
forms, only to the extent which we need in this article. In subsection 1.2, we define various Selmer group involved. In section 2, we prove a control theorem for Hilbert modular form and deduce Theorem 2.10. In section 3, we discuss the specialization results connecting the 'big' Selmer groups with the Selmer groups of the individual Hilbert modular forms at the fibres and prove the main theorem in the Hilbert modular form case (Theorem 3.10). We prove the second version of the main theorem for elliptic modular forms over $\mathbb{Z}_{p}^{2}$ extension in section 4 (Theorem 4.9).

Acknowledgment : We would like to thank T. Ochiai, A. Raghuram, B. Baskar, R. Greenberg, A. Pal and R. Sujatha for discussions. During this project, first named author was initially supported by JSPS postdoctoral fellowship and later by DST Inspire Faculty Award grant. The second author was initially supported by ISI postdoctoral fellowship and later by IISER Pune postdoctoral fellowship.

\section{Preliminaries}

\subsection{Preliminary results on Hilbert Modular Forms.}

1.1.1. Hilbert Modular Forms and Nearly Ordinary Hecke Algebra. In this subsection, we collect some basic results about nearly ordinary Hida deformation of Hilbert modular forms and ordinary Hida deformation of elliptic modular forms, which are needed in the course of this article. All the results in this section are well known and can be found in the literature (cf. [Hi1], [Hi2], [Wi1], [Wi2]). Our presentation of results in this subsection, in many cases, follows the presentation of [F-O].

Let $p$ be an odd prime. Let $F$ be a totally real number field of degree $d, \mathcal{O}_{F}$ be the ring of integers of $F$, and $J_{F}$ denotes the set of embedding of $F$ into $\mathbb{R}$. To an ideal $\mathcal{M}$ of $\mathcal{O}_{F}$, we attach standard compact open subgroups $K_{0}, K_{1}$ and $K_{11}$ of $G l_{2}\left(\mathcal{O}_{F} \otimes_{\mathbb{Z}} \hat{\mathbb{Z}}\right)$ as follows:

$$
\begin{gathered}
K_{0}(\mathcal{M})=\left\{\left(\begin{array}{ll}
a & b \\
c & d
\end{array}\right) \in G l_{2}\left(\mathcal{O}_{F} \otimes_{\mathbb{Z}} \hat{\mathbb{Z}}\right) \mid c \equiv 0(\bmod \mathcal{M})\right\} \\
K_{1}(\mathcal{M})=\left\{\left(\begin{array}{ll}
a & b \\
c & d
\end{array}\right) \in K_{0}(\mathcal{M}) \mid d \equiv 1(\bmod \mathcal{M})\right\} \\
K_{11}(\mathcal{M})=\left\{\left(\begin{array}{ll}
a & b \\
c & d
\end{array}\right) \in K_{0}(\mathcal{M}) \mid a, d \equiv 1(\bmod \mathcal{M})\right\} .
\end{gathered}
$$

Definition 1.1. A weight $k=\sum_{\tau \in J_{F}} k_{\tau} \tau$ is an element of $\mathbb{Z}\left[J_{F}\right]$, an arithmetic weight is a weight such that $k_{\tau} \geq 2$ for all $\tau \in J_{F}$ and $k_{\tau}$ has constant parity. A parallel weight is an integral multiple of the weight $t=\sum_{\tau \in J_{F}} \tau$. Two weights are said to be equivalent if their deference is a parallel weight. To an arithmetic weight $k$, one associates a weight $v \in \mathbb{Z}\left[J_{F}\right]$, called the parallel defect of $k$, which satisfies $k+2 v \in \mathbb{Z} t$.

Let $\mathcal{O}$ be the ring of integers of a finite extension of $\mathbb{Q}_{p}$ which contains all conjugates of $F$. For $k$ an arithmetic weight and $v$ its parallel defect, $S_{k, w}(U ; \mathcal{O})$ denotes the holomorphic cusp forms of weight $(k, w)$ of level $U$ and coefficient in $\mathcal{O}$, where $U$ is a finite index subgroup of $G l_{2}\left(\mathcal{O}_{F} \otimes_{\mathbb{Z}} \hat{\mathbb{Z}}\right)$ containing $K_{11}(\mathcal{M})$ for some $\mathcal{M} \subset \mathcal{O}_{F}$, 
and $w=k+v-t$. A cuspidal Hilbert modular form $S_{k, w}(U ; \mathcal{O})$ is called primitive if it is not a Hilbert modular form of weight $(k, w)$ and of level smaller than $U$. A normalized primitive Hilbert eigenform is called a Hilbert newform. To $f \in S_{k, w}(U ; \mathcal{O})$, one can naturally associate an automorphic representation $\pi_{f} \in G l_{2}\left(\mathbb{A}_{F}\right)$.

Fix an ideal $\mathcal{N}$ of $F$ which is prime to p and for any $s \in \mathbb{N}$, we have an action of $G=\left(\mathcal{O}_{F} \otimes_{\mathbb{Z}} \mathbb{Z}_{p}\right)^{*} \times\left(\left(\mathcal{O}_{F} \otimes_{\mathbb{Z}} \mathbb{Z}_{p}\right)^{*} / \overline{\mathcal{O}}_{F}^{*}\right)$ on $S_{k, w}\left(K_{1}(\mathcal{N}) \cap K_{11}\left(p^{s}\right) ; \mathcal{O}\right)$. We have an action of the $p$-Hecke operator $T_{0}(p)$, normalized according to the parallel defect $v$, on the space $S_{k, w}\left(K_{1}(\mathcal{N}) \cap K_{11}\left(p^{s}\right) ; \mathcal{O}\right)$. The largest $\mathcal{O}$ submodule of $S_{k, w}\left(K_{1}(\mathcal{N}) \cap\right.$ $\left.K_{11}\left(p^{s}\right) ; \mathcal{O}\right)$ on which $T_{0}(p)$ acts invertibly is denoted by $S_{k, w}^{n . o .}\left(K_{1}(\mathcal{N}) \cap K_{11}\left(p^{s}\right) ; \mathcal{O}\right)$. A form $f \in S_{k, w}\left(K_{1}(\mathcal{N}) \cap K_{11}\left(p^{s}\right) ; \mathcal{O}\right)$ is called nearly ordinary if $f \in S_{k, w}^{n . o .}\left(K_{1}(\mathcal{N}) \cap\right.$ $\left.K_{11}\left(p^{s}\right) ; \mathcal{O}\right)$.

The nearly ordinary Hecke algebra $\mathbf{H}_{k, w}\left(K_{1}(\mathcal{N}) \cap K_{11}\left(p^{s}\right) ; \mathcal{O}\right)$ of weight $(k, w)$ and level $K_{1}(\mathcal{N}) \cap K_{11}\left(p^{s}\right)$ is defined to be the $\mathcal{O}$ subalgebra of $\operatorname{End}_{\mathcal{O}}\left(S_{k, w}^{n . o .}\left(K_{1}(\mathcal{N}) \cap\right.\right.$ $\left.K_{11}\left(p^{s}\right) ; \mathcal{O}\right)$ ) generated by the Hecke operators. The $\mathcal{O}$ algebra $\mathbf{H}_{k, w}\left(K_{1}(\mathcal{N}) \cap\right.$ $\left.K_{11}\left(p^{s}\right) ; \mathcal{O}\right)$ is finite flat over $\mathcal{O}$.

Let $k$ be an arithmetic weight and $v$ be its parallel defect. By the perfect duality between $\mathbf{H}_{k, w}\left(K_{1}(\mathcal{N}) \cap K_{11}\left(p^{s}\right) ; \mathcal{O}\right)$ and $S_{k, w}^{n . o .}\left(K_{1}(\mathcal{N}) \cap K_{11}\left(p^{s}\right) ; \mathcal{O}\right)$, giving an eigen cuspform $f \in S_{k, w}^{n . o .}\left(K_{1}(\mathcal{N}) \cap K_{11}\left(p^{s}\right) ; \mathcal{O}\right)$ is equivalent to giving an algebra homomorphism

$$
q_{f}: \mathbf{H}_{k, w}\left(K_{1}(\mathcal{N}) \cap K_{11}\left(p^{s}\right) ; \mathcal{O}\right) \rightarrow \mathbf{H}_{k, w}\left(K_{1}\left(\mathcal{N} p^{s}\right) ; \mathcal{O}\right) \rightarrow \overline{\mathbb{Q}}_{p}
$$

sending $T \in \mathbf{H}_{k, w}\left(K_{1}\left(\mathcal{N} p^{s}\right) ; \mathcal{O}\right)$ to $a_{1}(f \mid T)$.

Let $\Lambda_{\mathcal{O}}$ denote the completed group algebra $\mathcal{O}\left[G / G_{\text {tors }}\right]$. The algebra $\Lambda_{\mathcal{O}}$ is noncanonically isomorphic to the power series algebra $\mathcal{O}\left[\left[X_{1}, \cdots, X_{r}\right]\right]$, where $r=1+$ $d+\delta_{F, p}, \delta_{F, p}$ be the defect of the Leopoldt's conjecture for $F$ at $p$.

Let the nearly ordinary Hecke algebra $\mathbf{H}_{\mathcal{N}, \mathcal{O}}$ be the inverse limit w.r.t. $s$ of the $\mathbf{H}_{2 t, 0}\left(K_{1}(\mathcal{N}) \cap K_{11}\left(p^{s}\right) ; \mathcal{O}\right)$. By fundamental work of Hida, for any arithmetic weight $k \in \mathbb{Z}\left[J_{F}\right]$, we have $\mathbf{H}_{\mathcal{N}, \mathcal{O}} \cong \underbrace{}_{s} \mathbf{H}_{k, w}\left(K_{1}(\mathcal{N}) \cap K_{11}\left(p^{s}\right) ; \mathcal{O}\right)$. The nearly ordinary Hecke algebra $\mathbf{H}_{\mathcal{N}, \mathcal{O}}$ is finite torsion free $\Lambda_{\mathcal{O}}$ module and hence a semi-local ring. Let $\mathfrak{a}$ be one of the finitely many ideals of height zero in $\mathbf{H}_{\mathcal{N}, \mathcal{O}}$. The algebra $\mathcal{R}=\mathbf{H}_{\mathcal{N}, \mathcal{O}} / \mathfrak{a}$ is called a branch of $\mathbf{H}_{\mathcal{N}, \mathcal{O}}$.

Definition 1.2. For a weight $k \in \mathbb{Z}\left[J_{F}\right]$, an algebraic character $\xi: G=\left(\mathcal{O}_{F} \otimes_{\mathbb{Z}}\right.$ $\left.\mathbb{Z}_{p}\right)^{*} \times\left(\left(\mathcal{O}_{F} \otimes_{\mathbb{Z}} \mathbb{Z}_{p}\right)^{*} / \overline{\mathcal{O}}_{F}^{*}\right) \rightarrow \overline{\mathbb{Q}}_{p}^{*}$ of weight $(k, w)$ is a character of the form, $\xi(a, z)=$ $\psi(a, z) \chi_{\text {cyc }}^{[n+2 v]}(z) a^{n}$, where $\psi$ is a character of finite order, $[n+2 v]$ is the unique integer satisfying $n+2 v=[n+2 v] t$ (Recall, $n=k+2 t, w=k+v-t)$. An algebraic character of weight $(k, w)$ is an arithmetic character of weight $(k, w)$ if its restriction to $\mathcal{O}_{F}^{*} \subset\left(\mathcal{O}_{F} \otimes_{\mathbb{Z}} \mathbb{Z}_{p}\right)^{*}$ is trivial. An algebra homomorphism, $\xi \in \operatorname{Hom}\left(\Lambda_{\mathcal{O}}, \overline{\mathbb{Q}}_{p}\right)$ is algebraic (resp. arithmetic) of weight $(k, w)$ if $\left.\xi\right|_{G}$ is algebraic (resp. arithmetic) of weight $(k, w)$. If $R$ is a finite $\Lambda_{\mathcal{O}}$ algebra, $\xi \in \operatorname{Hom}\left(R, \overline{\mathbb{Q}}_{p}\right)$ is algebraic (resp. arithmetic) of weight $(k, w)$ if $\left.\xi\right|_{\Lambda_{\mathcal{O}}}$ is algebraic (resp. arithmetic) of weight $(k, w)$. A prime ideal $P_{\xi} \subset R$ which is defined as the kernel of an algebraic (resp. arithmetic) specialization of $\xi$ of $R$ is called an algebraic (resp. arithmetic) point. 
For any $k$ be an arithmetic weight and any of its parallel defect $v$, and for any nearly ordinary eigen cuspform $f \in S_{k, w}^{n . o .}\left(K_{1}(\mathcal{N}) \cap K_{11}\left(p^{s}\right) ; \mathcal{O}\right)$ which is new at every prime diving $\mathcal{N}$, there exists a unique branch $\mathcal{R}$ of $\mathbf{H}_{\mathcal{N}, \mathcal{O}}$ and a unique arithmetic specialization $\xi_{f}: \mathcal{R} \rightarrow \overline{\mathbb{Q}}_{p}$ of weight $(k, w)$ such that $\xi_{f}(\mathcal{R})$ is canonically identified with $q_{f}\left(\mathbf{H}_{k, w}\left(K_{1}(\mathcal{N}) \cap K_{11}\left(p^{s}\right) ; \mathcal{O}\right)\right)$.

Let $\mathcal{R}$ be a branch of $\mathbf{H}_{\mathcal{N}, \mathcal{O}}$. Then for any $k$ be an arithmetic weight and any of its parallel defect $v$, and for any arithmetic specialization $\xi: \mathcal{R} \rightarrow \overline{\mathbb{Q}}_{p}$ of weight $(k, w)$, there exists a unique nearly ordinary eigen cuspform $f_{\xi} \in \mathbf{H}_{k, w}\left(K_{1}\left(\mathcal{N} p^{s}\right) ; \mathcal{O}\right)$ for some $s$, such that $\xi(\mathcal{R})$ is canonically identified with $q_{f_{\xi}}\left(\mathbf{H}_{k, w}\left(K_{1}(\mathcal{N}) \cap K_{11}\left(p^{s}\right) ; \mathcal{O}\right)\right)$. We will donate the set of arithmetic points of $\mathcal{R}$ by $\mathfrak{X}(\mathcal{R})$. For each $\xi \in \mathfrak{X}(\mathcal{R})$, $P_{\xi}=\operatorname{ker}(\xi)$ is a coheight 1 prime ideal in $\mathcal{R}$.

1.1.2. Galois Representation. Galois representation associated to a Hilbert modular eigen cuspform was constructed and studied by Carayol, Ohta, Wiles, Taylor and Blasius-Rogawski. We briefly recall their results in the following two theorems.

Theorem 1.3. Let $f \in S_{k, w}\left(K_{1}(\mathcal{M}) ; \overline{\mathbb{Q}}_{p}\right)$ be a normalized eigen cuspform of arithmetic weight $k$, and let $K$ be a finite extension of $\mathbb{Q}_{p}$ containing all Hecke eigenvalues for $f$. Then there exists a continuous irreducible $G_{F}$ representation $V_{f} \cong K^{\oplus 2}$, which is unramified outside $\mathcal{M p}$ and satisfies

$$
\operatorname{det}\left(1-F r_{\lambda} X \mid V_{f}\right)=1-T_{\lambda}(f) X+S_{\lambda}(f) X^{2}
$$

for all $\lambda \nmid \mathcal{M} p$, where $T_{\lambda}$ (resp. $S_{\lambda}$ ) is the Hecke operator induced by the coset class $K_{1}(\mathcal{M})\left(\begin{array}{cc}1 & 0 \\ 0 & \pi_{\lambda}\end{array}\right) K_{1}(\mathcal{M})$ (resp. $K_{1}(\mathcal{M})\left(\begin{array}{cc}\pi_{\lambda} & 0 \\ 0 & \pi_{\lambda}\end{array}\right) K_{1}(\mathcal{M})$ ), where $\pi_{\lambda}$ is a uniformizer at $\lambda$ and $F r_{\lambda}$ is the geometric Frobenius at $\lambda$.

The $G_{F}$ representation $V_{f}$ is known to be irreducible, and hence characterized upto isomorphism by the above equation.

Remark 1.4. Let $f \in S_{k, w}\left(K_{1}(\mathcal{M}) ; \overline{\mathbb{Q}}_{p}\right)$ be a normalized eigen cuspform of arithmetic weight $k$, and let $K$ be a finite extension of $\mathbb{Q}_{p}$ containing all Hecke eigenvalues for $f$ as in Theorem 1.3. If $\mathfrak{p} \mid p$, let $c(\mathfrak{p}, f)$ be the $T(\mathfrak{p})$ eigenvalue of $f$. We say $f$ is ordinary at $\mathfrak{p}$ if $c(\mathfrak{p}, f)$ is a unit in the ring of integers of $K$ and $f$ is ordinary at $p$ if and only if for all $\mathfrak{p} \mid p, f$ is ordinary at $\mathfrak{p}$.

Next theorem describes the local properties of the Galois representation $V_{f}$.

Theorem 1.5. Let $f \in S_{k, w}\left(K_{1}(\mathcal{M}) ; \overline{\mathbb{Q}}_{p}\right)$ be a normalized eigen cuspform. Let $w_{\max }=\max \left\{w_{\tau}, \tau \in J_{F}\right\}$. Let $V_{f}$ (resp. $\pi_{f}$ ) be the Galois representation (resp. automorphic representation) associated to $f$.

(1) If $\lambda \nmid p$, then

(a) The inertia group $I_{\lambda}$ at $\lambda$ acts on $V_{f}$ through infinite quotient iff $\pi_{f, \lambda}$ is a Steinberg representation. In this case, $V_{f}$ has a unique filtration by graded pieces of dimension one:

$$
0 \rightarrow\left(V_{f}\right)_{\lambda}^{+} \rightarrow V_{f} \rightarrow\left(V_{f}\right)_{\lambda}^{-} \rightarrow 0
$$

which is stable under the decomposition group $G_{\lambda}$ at $\lambda$. The inertia group $I_{\lambda}$ acts on $\left(V_{f}\right)_{\lambda}^{+}$(resp. $\left.\left(V_{f}\right)_{\lambda}^{-}\right)$through a finite quotient of $I_{\lambda}$. 
An eigenvalue $\alpha$ of the action of a lift of $F r_{\lambda}$ to $G_{\lambda}$ on $\left(V_{f}\right)_{\lambda}^{+}$(resp. $\left.\left(V_{f}\right)_{\lambda}^{-}\right)$is an algebraic number satisfying $|\alpha|_{\infty}=\left(N_{F / \mathbb{Q}}(\lambda)\right)^{\frac{w_{\max }+1}{2}}$ (resp. $\left.\left(N_{F / \mathbb{Q}}(\lambda)\right)^{\frac{w_{\max }-1}{2}}\right)$.

(b) If $I_{\lambda}$ acts on $V_{f}$ through a finite quotient, the action of $I_{\lambda}$ is reducible iff $\pi_{f, \lambda}$ is principal series. If $I_{\lambda}$ acts on $V_{f}$ through a finite quotient, an eigenvalue $\alpha$ of the action of a lift of $F r_{\lambda}$ to $G_{\lambda}$ on $V_{f}$ is an algebraic number satisfying $|\alpha|_{\infty}=\left(N_{F / \mathbb{Q}}(\lambda)\right)^{\frac{w_{\max }}{2}}$.

(2) If $\mathfrak{p} \mid p$, and if $f$ is nearly ordinary at $\mathfrak{p}$. Then $V_{f}$ has a unique filtration by graded pieces of dimension one:

$$
0 \rightarrow\left(V_{f}\right)_{\mathfrak{p}}^{+} \rightarrow V_{f} \rightarrow\left(V_{f}\right)_{\mathfrak{p}}^{-} \rightarrow 0
$$

which is stable under the decomposition group $G_{\mathfrak{p}}$ at $\mathfrak{p}$, and Hodge-Tate weight of $\left(V_{f}\right)_{\mathfrak{p}}^{+}$is greater then Hodge-Tate weight of $\left(V_{f}\right)_{\mathfrak{p}}^{-}$.

Remark 1.6. Let $f \in S_{k, w}\left(K_{1}(\mathcal{M}) ; \overline{\mathbb{Q}}_{p}\right)$ be a normalized eigen cuspform of arithmetic weight $k$ with associated Galois representation $V_{f}$ over $K$ as in Theorem 1.3. If $f$ is p-ordinary, then for all primes $\mathfrak{p} \mid p$,

$$
\left.V_{f}\right|_{G_{\mathfrak{p}}} \sim\left[\begin{array}{cc}
\epsilon_{\mathfrak{p}} & * \\
0 & \delta_{\mathfrak{p}}
\end{array}\right],
$$

where $\epsilon_{\mathfrak{p}}, \delta_{\mathfrak{p}}$ are characters of $G_{\mathfrak{p}}$ with values in $K^{*}$ and $\delta_{\mathfrak{p}}$ is unramified. In the case of nearly ordinary $f$ at $\mathfrak{p}$, our Galois representation restricted $G_{\mathfrak{p}}$ looks same, except $\delta_{\mathfrak{p}}$ need not be unramified.

The following two theorems are the Hida family versions of the two above theorem, and are due to the work of Wiles and Hida.

Theorem 1.7. Let $\mathcal{R}$ be a branch of $\mathbf{H}_{\mathcal{N}, \mathcal{O}}$. Then there exists a finitely generated torsion free $\mathcal{R}$ module $\mathcal{T}_{\mathcal{R}}$ with continuous $G_{F}$ action, which satisfies the following properties:

(1) The vector space $\mathcal{V}_{\mathcal{R}}:=\mathcal{T}_{\mathcal{R}} \otimes_{\mathcal{R}} \mathcal{K}$ is of dimension 2 over $\mathcal{K}$, where $\mathcal{K}$ is the fraction field of $\mathcal{R}$.

(2) The representation $\rho_{\mathcal{R}}$ of $G_{F}$ on $\mathcal{V}_{\mathcal{R}}$ is irreducible and unramified outside primes dividing $\mathcal{N} p \infty$.

(3) For any arithmetic weight $(k, w)$, and for any nearly ordinary eigen cuspform $f \in S_{k, w}^{n . o}\left(K_{1}(\mathcal{N}) \cap K_{11}\left(p^{s}\right) ; \mathcal{O}\right)$ which corresponds to the arithmetic weight $\xi=$ $\xi_{f}$ on the branch $\mathcal{R}, T_{f}=\mathcal{T}_{\mathcal{R}} \otimes_{\mathcal{R}} \xi_{f}(\mathcal{R})$ is a lattice of the Galois representation $V_{f}$ associated to $f$.

Next theorem characterizes the local behavior of the Galois representation associated to $\mathcal{R}$.

Theorem 1.8. Let $\mathcal{R}$ be a branch of $\mathbf{H}_{\mathcal{N}, \mathcal{O}}$ and $\mathcal{V}_{\mathcal{R}}=\mathcal{V}$ be the Galois representation over $\mathcal{K}$. Then,

(1) For every prime $\lambda \nmid \mathcal{N} p$, we have:

$$
\operatorname{det}\left(1-F r_{\lambda} X \mid \mathcal{V}\right) \underset{8}{=1}-T_{\lambda} X+S_{\lambda} X^{2}
$$


where $T_{\lambda}$ and $S_{\lambda}$ are Hecke operators on $\mathcal{R}$ at $\lambda$ which is obtained at the limit of the Hecke operators in Theorem 1.3 at finite levels.

(2) For every prime $\mathfrak{p} \mid p$, we have a canonical filtration obtained as the limit of the filtration given in Theorem 1.5:

$$
0 \rightarrow \mathcal{V}_{\mathfrak{p}}^{+} \rightarrow \mathcal{V} \rightarrow \mathcal{V}_{\mathfrak{p}}^{-} \rightarrow 0
$$

which is stable under the action of the decomposition group $G_{\mathfrak{p}}$ at $\mathfrak{p}$.

For an ideal $\mathcal{N}$ in $\mathcal{O}_{F}$ which is prime to $p$, we have a Hida's nearly ordinary Hecke algebra $\mathbf{H}_{\mathcal{N}, \mathcal{O}}$. We fix a branch $\mathcal{R}$ of $\mathbf{H}_{\mathcal{N}, \mathcal{O}}$ and a representation $\mathcal{T}=\mathcal{T}_{\mathcal{R}}$ as in Theorem 1.7. We assume that we have a $G_{\mathfrak{p}}$ stable filtration

$$
0 \rightarrow \mathcal{T}_{\mathfrak{p}}^{+} \rightarrow \mathcal{T} \rightarrow \mathcal{T}_{\mathfrak{p}}^{-} \rightarrow 0
$$

by finite type $\mathcal{R}$ modules $\mathcal{T}_{\mathfrak{p}}^{+}$and $\mathcal{T}_{\mathfrak{p}}^{-}$with continuous $G_{\mathfrak{p}}$ action which gives the exact sequence

$$
0 \rightarrow \mathcal{V}_{\mathfrak{p}}^{+} \rightarrow \mathcal{V} \rightarrow \mathcal{V}_{\mathfrak{p}}^{-} \rightarrow 0
$$

in Theorem 1.8 by taking base extension to $\mathcal{K}$.

Let $\mathfrak{m}_{\mathcal{R}}$ denote the maximal ideal of $\mathcal{R}$ and $\mathbb{F}_{\mathcal{R}}$ be the finite field $\mathcal{R} / \mathfrak{m}_{\mathcal{R}}$. Associated to the Galois representation of $G_{F}$ in Theorem 1.7, there exist a canonical residual Galois representation $\bar{\rho}_{\mathcal{R}}: G_{F} \longrightarrow G L_{2}\left(\mathbb{F}_{\mathcal{R}}\right)$. Throughout this article, we assume the following two hypotheses on this $\bar{\rho}$.

Hypothesis 1.9. (Irr): The residual representation $\bar{\rho}_{\mathcal{R}}$ of $G_{F}$ is absolutely irreducible.

Hypothesis 1.10. (Dist): As before, let $G_{\mathfrak{p}}$ be the decomposition subgroup of $G_{F}$ at $\mathfrak{p}$. The restriction of the residual representation at the decomposition subgroup i.e. $\left.\bar{\rho}_{\mathcal{R}}\right|_{G_{\mathfrak{p}}}$ is an extension of two distinct characters of $G_{\mathfrak{p}}$ with values in $\mathbb{F}_{\mathcal{R}}^{*}$ for each $\mathfrak{p} \mid p$.

Remark 1.11. Conditions (Irr) and (Dist) together implies that the representation $\mathcal{T}$ can be chosen to be free of rank two over $\mathcal{R}$ and, for each $\mathfrak{p} \mid p$, the graded pieces $\mathcal{T}_{\mathfrak{p}}^{+}$and $\mathcal{T}_{\mathfrak{p}}^{-}$are both free of rank one over $\mathcal{R}$.

1.1.3. ordinary Hida deformation of elliptic modular forms. We summarize the relevant results for $p$-ordinary Hida family of elliptic modular forms in the following remark. This details can be found in the literature (also in [J-P, Section 1]).

Remark 1.12. Let $f=\sum a_{n} q^{n}$ be a normalized elliptic eigenform of weight $k \geq 2$, level $N$ and nebentypus $\psi$. We say that $f$ is p-ordinary if $\iota_{p}\left(a_{p}\right)$ is a p-adic unit. Also assume that $f$ is p-stabilized (p-refined). By the work of Eichler-Shimura, Deligne, Mazur-Wiles, Wiles and many other people, to such an $f$, one can associate a Galois representation $\rho_{f}: G_{\mathbb{Q}} \rightarrow G L\left(V_{f}\right)$, here $V_{f}$ is a two dimensional vector space over a finite extension of $\mathbb{Q}_{p}$, which is unramified outside $N p$, and for any prime $l \nmid N p$, arithmetic Frobl has the characteristic polynomial $X^{2}-a_{l} X+\psi(l) l^{k-1}$, moreover, restricted to the decomposition subgroup at $p$, we have, $\left.V_{f}\right|_{G_{p}} \sim\left[\begin{array}{cc}\epsilon_{1} & * \\ 0 & \epsilon_{2}\end{array}\right]$ with $\epsilon_{2}$ unramified. We have a notion of Hida family and arithmetic points for elliptic 
modular forms of tame level $N$. A theorem of Hida asserts that if $(N, p)=1$ and $f$ is a p-stabilized newform of weight $k \geq 2$, tame level $N$, then there exists a branch $\mathcal{R}$ of an ordinary Hida family and an arithmetic point $\xi: \mathcal{R} \longrightarrow \overline{\mathbb{Q}}_{p}$ such that $\xi(\mathcal{R})$ canonically corresponds with $f$. By the work of Hida and Wiles, to $\mathcal{R}$ one can associate a big Galois representation of dimension two, $\rho_{\mathcal{R}}: G_{\mathbb{Q}} \rightarrow G L\left(\mathcal{V}_{\mathcal{R}}\right)$, where $\mathcal{V}_{\mathcal{R}}$ is a vector space of dimension two over $\mathcal{K}$, the fraction field of $\mathcal{R}$. The Galois representation $\rho_{\mathcal{R}}$ is unramified at all finite places outside $N p$ and for a prime $l \nmid N p$, one has $\operatorname{det}\left(1-\left.F r_{l} X\right|_{\mathcal{V}}\right)=1-T_{l} X+S_{l} X^{2}$, where $T_{l}, S_{l}$ are Hecke operators on $\mathcal{R}$ at $l$, moreover, $\left.\mathcal{V}_{\mathcal{R}}\right|_{G_{p}} \sim\left[\begin{array}{cc}\tilde{\epsilon}_{1} & * \\ 0 & \tilde{\epsilon}_{2}\end{array}\right]$ with $\tilde{\epsilon}_{2}$ unramified. Similar to the case of nearly ordinary Hilbert modular forms, throughout the article, we make the following two hypotheses.

Hypothesis 1.13. (Irr): The residual representation $\bar{\rho}_{\mathcal{R}}$ of $G_{\mathbb{Q}}$ is absolutely irreducible.

Hypothesis 1.14. (Dist):For the representation $\left.\bar{\rho}_{\mathcal{R}}\right|_{G_{p}}$, we have $\tilde{\epsilon}_{1} \neq \tilde{\epsilon}_{2}\left(\bmod \mathfrak{m}_{\mathcal{R}}\right)$.

Under (Irr) and (Dist), we have the lattices $T_{f}$ in $V_{f}$ and $\mathcal{T}_{\mathcal{R}}$ in $\mathcal{V}_{\mathcal{R}}$ invariant under $\rho_{f}$ and $\rho_{\mathcal{R}}$ respectively, such that $T_{f} \cong \mathcal{T}_{\mathcal{R}} \otimes_{\mathcal{R}} \xi(\mathcal{R})$. Moreover, $\mathcal{T}_{\mathcal{R}}$ has a filtration as $G_{p}$ module,

$$
0 \rightarrow \mathcal{T}_{\mathcal{R}}^{+} \rightarrow \mathcal{T}_{\mathcal{R}} \rightarrow \mathcal{T}_{\mathcal{R}}^{-} \rightarrow 0
$$

such that the graded pieces $\mathcal{T}_{\mathcal{R}}^{+}$and $\mathcal{T}_{\mathcal{R}}^{-}$are free $\mathcal{R}$ module of rank one.

1.2. Various Selmer groups. We fix a totally real number field $F$ and as $S=S_{F}$ is a finite set of finite places of $F$ containing the primes lying above $\mathcal{N} p$. Let $f \in S_{k, w}^{n . o .}\left(K_{1}(\mathcal{N}) \cap K_{11}\left(p^{s}\right) ; \mathcal{O}\right)$. Let $V_{f}$ denote the Galois representation associated to $f$ over $K_{f}$, a finite extension of $\mathbb{Q}_{p}$ containing all Hecke eigenvalues of $f$ as in Theorem 1.3. We denote the ring of integer of $K_{f}$ by $O_{f}$. Let $T_{f} \subset V_{f}$ be a $G_{F}$ invariant lattice. Thus we have an induced action of $G_{F}$ on the discrete module $A_{f}:=V_{f} / T_{f}$. Further, since $\rho_{f}$ is nearly ordinary at $p, A_{f}$ has a filtration as a $G_{\mathfrak{p}}$ module for every $\mathfrak{p} \mid p$,

$$
0 \longrightarrow\left(A_{f}\right)_{\mathfrak{p}}^{+} \longrightarrow A_{f} \longrightarrow\left(A_{f}\right)_{\mathfrak{p}}^{-} \longrightarrow 0,
$$

where both $\left(A_{f}\right)_{\mathfrak{p}}^{+\vee}$ and $\left(A_{f}\right)_{\mathfrak{p}}^{-\vee}$ are free over $O_{f}$ of rank 1 .

Let $\mathcal{L}$ be an finite or infinite algebraic extension of $F$ and $w$ will denote a prime in $\mathcal{L}$. The notation $w \mid S$ will mean the prime $w$ of $\mathcal{L}$ is lying above a prime in $S$. Given such a prime $w$, let $G_{w}$ and $I_{w}$ respectively denote the decomposition subgroup and inertia subgroup for the extension $\overline{\mathbb{Q}} / \mathcal{L}$ with respect to the primes $\bar{w} / w$, where we have fixed a prime $\bar{w}$ of $\overline{\mathbb{Q}}$ over $w$. We denote the Frobenius element at $w$ by $\operatorname{Fr}_{w}$ so that $G_{w} / I_{w} \cong<\operatorname{Fr}_{w}>$.

Let $\mathcal{R}$ be a branch of $\mathbf{H}_{\mathcal{N}, \mathcal{O}}$. By Theorem 1.7, we have a free $\mathcal{R}$ lattice $\mathcal{T}=\mathcal{T}_{\mathcal{R}}$. Define,

$$
\mathcal{A}=\mathcal{A}_{\mathcal{R}}:=\mathcal{T} \otimes_{\mathcal{R}} \operatorname{Hom}_{\text {cont }}\left(\mathcal{R}, \mathbb{Q}_{p} / \mathbb{Z}_{p}\right)
$$

For any arithmetic character $\xi$ of $\mathcal{R}$, we have from definition $1.2, \mathcal{A}\left[P_{\xi}\right] \cong A_{f_{\xi}}$. Using Theorem 1.8 and by the assumption 1.10 , we get $\mathcal{A}$ has a filtration as a $G_{\mathfrak{p}}$ module

$$
0 \longrightarrow \mathcal{A}_{\mathfrak{p}}^{+} \longrightarrow \mathcal{A} \longrightarrow \mathcal{A}_{\mathfrak{p}}^{-} \longrightarrow 0
$$


where both $\mathcal{A}_{\mathfrak{p}}^{+\vee}$ and $\mathcal{A}_{\mathfrak{p}}^{-\vee}$ are free $\mathcal{R}$ modules of rank 1 . Also we have $\mathcal{A}_{\mathfrak{p}}^{-}\left[P_{\xi}\right] \cong$ $\left(A_{f_{\xi}}\right)_{\mathfrak{p}}^{-}$.

Let $L$ be a finite extension of $K$. We define various Selmer groups associated to $f$ and $\mathcal{R}$, defined over $L$.

Definition 1.15 (Greenberg Selmer group of $f$ ).

$$
S\left(A_{f} / L\right)=\operatorname{ker}\left(H^{1}\left(F_{S} / L, A_{f}\right) \longrightarrow \underset{w \mid S, w \nmid p}{\bigoplus} H^{1}\left(I_{w}, A_{f}\right)^{G_{w}} \underset{w|\mathfrak{p}| p}{\bigoplus} H^{1}\left(I_{w},\left(A_{f}\right)_{\mathfrak{p}}^{-}\right)^{G_{w}}\right)
$$

Definition 1.16 (Strict Selmer group of $f$ ).

$$
S^{\prime}\left(A_{f} / L\right):=\operatorname{ker}\left(H^{1}\left(F_{S} / L, A_{f}\right) \longrightarrow \underset{w \mid S, w \nmid p}{\oplus} H^{1}\left(I_{w}, A_{f}\right)^{G_{w}} \underset{w|\mathfrak{p}| p}{\oplus} H^{1}\left(G_{w},\left(A_{f}\right)_{\mathfrak{p}}^{-}\right)\right)
$$

Definition 1.17 (Greenberg Selmer group of $\mathcal{R}$ ).

$$
S(\mathcal{A} / L)=\operatorname{ker}\left(H^{1}\left(F_{S} / L, \mathcal{A}\right) \longrightarrow \underset{w \mid S, w \nmid p}{\bigoplus} H^{1}\left(I_{w}, \mathcal{A}\right)^{G_{w}} \underset{w|\mathfrak{p}| p}{\oplus} H^{1}\left(I_{w}, \mathcal{A}_{\mathfrak{p}}^{-}\right)^{G_{w}}\right)
$$

Definition 1.18 (Strict Selmer group of $\mathcal{R}$ ).

$$
S^{\prime}(\mathcal{A} / L):=\operatorname{ker}\left(H^{1}\left(F_{S} / L, \mathcal{A}\right) \longrightarrow \underset{w \mid S, w \nmid p}{\bigoplus} H^{1}\left(I_{w}, \mathcal{A}\right)^{G_{w}} \underset{w|\mathfrak{p}| p}{\bigoplus} H^{1}\left(G_{w}, \mathcal{A}_{\mathfrak{p}}^{-}\right)\right)
$$

Definition 1.19. Let $S^{\perp} \in\left\{S, S^{\prime}\right\}$. Define the Pontryagin dual

$$
X^{\perp}\left(T_{f} / L\right)=H_{\text {cont }}\left(S^{\perp}\left(A_{f} / L\right), \mathbb{Q}_{p} / \mathbb{Z}_{p}\right)
$$

where $X^{\perp}=X$ if $S^{\perp}=S$ and $X^{\perp}=X^{\prime}$ if $S^{\perp}=S^{\prime}$.

Define $\mathcal{X}^{\perp}\left(\mathcal{T}_{\mathcal{R}} / L\right)$ by replacing $X^{\perp}$ by $\mathcal{X}^{\perp}, A_{f}$ by $\mathcal{A}$ and $T_{f}$ with $\mathcal{T}_{\mathcal{R}}$ in (13).

For an infinite extension $F_{\infty}$ of $F$, the Selmer group $S^{\perp}\left(A_{f} / F_{\infty}\right)$ (respectively $\left.S^{\perp}\left(\mathcal{A} / F_{\infty}\right)\right)$ is defined by taking the inductive limit of $S^{\perp}\left(A_{f} / L^{\prime}\right)\left(\operatorname{resp} . S^{\perp}\left(\mathcal{A} / L^{\prime}\right)\right)$ over all finite extensions $L^{\prime}$ of $F$ contained in $F_{\infty}$ with respect to the natural restriction maps. The corresponding Pontryagin duals are denoted by $X\left(T_{f} / F_{\infty}\right)$ and $\mathcal{X}\left(\mathcal{T}_{\mathcal{R}} / F_{\infty}\right)$ respectively.

Under the natural action of $\Gamma=\operatorname{Gal}\left(F_{\text {cyc }} / F\right), X\left(T_{f} / F_{\text {cyc }}\right)\left(\right.$ respectively $\mathcal{X}\left(\mathcal{T}_{\mathcal{R}} / F_{\text {cyc }}\right)$ ) acquires the structure of a $O_{f}[[\Gamma]]$ (respectively $\mathcal{R}[[\Gamma]]$ ) module. Also note that for $B \in\left\{A_{f}, \mathcal{A}\right\}$, we have $0 \longrightarrow S^{\prime}\left(B / F_{\text {cyc }}\right) \hookrightarrow S\left(B / F_{\text {cyc }}\right)$.

Next we discuss various types of twisted Selmer groups. For any $\mathbb{Z}_{p}$ module $M$, let $M(1)$ denotes the Tate twist of $M$ by the $p$-adic cyclotomic character $\chi_{p}: \Gamma \longrightarrow \mathbb{Z}_{p}^{\times}$. Define

$$
\mathcal{T}_{\mathcal{R}}^{*}:=\operatorname{Hom}_{\mathcal{R}}\left(\mathcal{T}_{\mathcal{R}}, \mathcal{R}(1)\right) .
$$

We have a corresponding filtration of $\mathcal{T}^{*}$ as a $G_{\mathfrak{p}}$ module

$$
0 \longrightarrow\left(\mathcal{T}_{\mathcal{R}}^{*}\right)_{\mathfrak{p}}^{+} \longrightarrow \mathcal{T}_{\mathcal{R}}^{*} \longrightarrow\left(\mathcal{T}_{\mathcal{R}}^{*}\right)_{\mathfrak{p}}^{-} \longrightarrow 0,
$$

where the graded pieces are defined as $\left(\mathcal{T}_{\mathcal{R}}^{*}\right)_{\mathfrak{p}}^{+}:=\operatorname{Hom}_{\mathcal{R}}\left(\left(\mathcal{T}_{\mathcal{R}}\right)_{\mathfrak{p}}^{-}, \mathcal{R}(1)\right)$ and $\left(\mathcal{T}_{\mathcal{R}}^{*}\right)_{\mathfrak{p}}^{-}:=$ $\operatorname{Hom}_{\mathcal{R}}\left(\left(\mathcal{T}_{\mathcal{R}}\right)_{\mathfrak{p}}^{+}, \mathcal{R}(1)\right)$. We can now define

$$
\mathcal{A}^{*}:=\mathcal{T}_{\mathcal{R}}^{*} \otimes_{\mathcal{R}} \operatorname{Hom}_{\text {cont }}\left(\mathcal{R}, \mathbb{Q}_{p} / \mathbb{Z}_{p}\right) .
$$

From the above discussion, we can get a filtration of $\mathcal{A}^{*}$ as in (8). 
Also, corresponding to a newform $f$, we define

$$
T_{f}^{*}:=\operatorname{Hom}_{O_{f}}\left(T_{f}, O_{f}(1)\right) .
$$

Then it is easy to see that the quotient $\mathcal{T}^{*} \otimes_{\mathcal{R}} \xi(\mathcal{R})$ is isomorphic to $T_{f_{\xi}}^{*}$. Also define $A_{f}^{*}=T_{f}^{*} \otimes \mathbb{Q}_{p} / \mathbb{Z}_{p}$. Then as in (7), there is a filtration

$$
0 \longrightarrow\left(A_{f}^{*}\right)_{\mathfrak{p}}^{+} \longrightarrow A_{f}^{*} \longrightarrow\left(A_{f}^{*}\right)_{\mathfrak{p}}^{-} \longrightarrow 0,
$$

with $\left(A_{f}^{*}\right)_{\mathfrak{p}}^{+\vee}$ and $\left(A_{f}^{*}\right)_{\mathfrak{p}}^{-\vee}$ are free $O_{f}$ module of rank 1 .

From the above discussions, by making obvious modifications in the definitions 1.15, 1.17 and 1.19, we can now define the Greenberg Selmer groups $S\left(A_{f}^{*} / \mathcal{L}\right)$, $S\left(\mathcal{A}^{*} / \mathcal{L}\right)$ and their respective Pontryagin duals $X\left(T_{f}^{*} / \mathcal{L}\right)$ and $\mathcal{X}\left(T_{\mathcal{R}}^{*} / \mathcal{L}\right)$ for any finite or infinite extension $\mathcal{L}$ of $F$.

Let $\rho: \Gamma \longrightarrow O_{f}^{\times}$be a character. Set $T_{\rho}=O_{f}(\rho)$, the $G_{F}$ module with underlying group $O_{f}$ and an $G_{F}$ action on it via $\rho$. Set $T_{f}(\rho)=T_{\rho} \otimes_{O_{f}} T_{f}, A_{f}(\rho)=T_{\rho} \otimes_{O_{f}} A_{f}$ and $\left(A_{f}\right)_{\mathfrak{p}}^{-}(\rho)=T_{\rho} \otimes_{O_{f}}\left(A_{f}\right)_{\mathfrak{p}}^{-}$with the diagonal action of $G_{F}$. Let $M$ be an $O_{f}[[\Gamma]]$ module. Define $M(\rho)=T_{\rho} \otimes_{O_{f}} M$ with $\gamma \in \Gamma$ acting by diagonal action. Applying $\otimes_{O_{f}} T_{\rho}$ to the filtration in (7), we get a filtration for $T_{\rho} \otimes A_{f}$. Proceeding in a way similar to the definition 1.15, define the Greenberg Selmer groups with respect to the 'twist' $\rho, S\left(A_{f} \otimes T_{\rho} / \mathcal{L}\right)$ and $X\left(T_{\rho} \otimes T_{f} / \mathcal{L}\right)$, for any extension $\mathcal{L}$ of $F$ (possibly infinite). As $\rho$ acts trivially on $G_{F_{\mathrm{cyc}}}$, we notice that

$$
X\left(T_{\rho} \otimes T_{f} / F_{\text {cyc }}\right) \cong X\left(T_{f} / F_{\text {cyc }}\right) \otimes T_{\rho^{-1}}
$$

In particular $X\left(T_{\rho} \otimes T_{f} / F_{\text {cyc }}\right)$ is a finitely generated torsion $O_{f}[[\Gamma]]$ module if and only if $X\left(T_{f} / F_{\text {cyc }}\right)$ is so.

Remark 1.20. Let $f$ be a newform nearly ordinary at $p$. Then we can express $T_{f}^{*} \cong T_{f^{*}} \otimes O_{f}\left(\chi_{p}^{t}\right)$ as Galois modules for some $t \in \mathbb{Z}$ and for some newform $f^{*}$ which is nearly ordinary at $p$, has the same level and weight as $f$ but possibly different character. Hence for any extension $\mathcal{L}$ of $F$, we deduce that $X\left(T_{\rho} \otimes T_{f}^{*} / \mathcal{L}\right) \cong$ $X\left(T_{\rho^{\prime}} \otimes T_{f^{*}} / \mathcal{L}\right)$ for certain character $\rho^{\prime}: \Gamma \longrightarrow O_{f}^{\times}$. In particular, $X\left(T_{f}^{*} / F_{\text {cyc }}\right) \cong$ $X\left(T_{f^{*}} / F_{\text {cyc }}\right) \otimes O_{f}(s)$ for some $s \in \mathbb{Z}$.

Remark 1.21. Selmer group for p-ordinary elliptic modular forms and for the corresponding Hida family:

Let $f \in S_{k}\left(\Gamma_{0}\left(N p^{r}\right), \psi\right)$ be a p-ordinary, $p$-stabilized (elliptic) newform. Then by remark 1.12, via its Galois representation, we can associate to $f$, a lattice $T_{f}$. Set $A_{f}=V_{f} / T_{f}$. Assume the conditions (Irr) and (Dist). Then, again by remark 1.12, to a branch $\mathcal{R}$ of a p-ordinary Hida family of elliptic modular forms of tame level $N$, we have a free $\mathcal{R}$ lattice $\mathcal{T}_{\mathcal{R}}$ and we can define $\mathcal{A}=\mathcal{A}_{\mathcal{R}}:=\mathcal{T} \otimes_{\mathcal{R}} \operatorname{Hom}_{\text {cont }}\left(\mathcal{R}, \mathbb{Q}_{p} / \mathbb{Z}_{p}\right)$. Then by p-ordinarity, both $A_{f}$ and $\mathcal{A}$ are equipped with canonical filtration as $G_{p}$ modules.

Let $K$ be an imaginary quadratic field and $K_{\mathrm{cyc}}$ and $K_{\infty}$ be respectively, the unique cyclotomic and $\mathbb{Z}_{p}^{2}$ extension of $K$. We assume that $p$ splits in $K$ and the discriminant $D_{K}$ is coprime to $N$. Let $S=S_{K}$ be a finite set of places of $K$ containing the primes dividing $N p$. Then proceeding in a similar way as in definitions 1.15 - 1.19, we can define the Greenberg Selmer groups and the strict Selmer group of $f$ and $\mathcal{R}$ 
over $K_{\mathrm{cyc}}$ and $K_{\infty}$. In fact, we will use the same symbols used in the above definitions. However, there is no case of confusion, as we deal with the elliptic modular forms and their ordinary Hida family only in section 4.

\section{FUNCTIONAL EQUATION FOR A HiLBERT MODULAR FORM}

A control theorem is a widely used tool in Iwasawa theory. We prove a 'control theorem' for the twisted Selmer group $X\left(T_{f} \otimes T_{\rho} / F_{\text {cyc }}\right)$ with $\rho$ as before. A control theorem in case of elliptic modular forms was discussed in [J-P, Theorem 3.1]. Recall, there is a tower of fields $F=F_{0} \subset \ldots \subset F_{n} \subset \ldots \subset F_{\text {cyc }}$ are such that $\operatorname{Gal}\left(F_{n} / F\right) \cong$ $\mathbb{Z} / p^{n} \mathbb{Z}$. Set $\Gamma_{n}=\operatorname{Gal}\left(F_{\text {cyc }} / F_{n}\right)$. Given a cuspidal Hilbert newform $f \in S_{k, w}^{n . o}\left(K_{1}(N) \cap\right.$ $\left.K_{11}\left(p^{s}\right) ; \mathcal{O}\right)$, let $\rho_{f}$ and $\pi_{f}$ respectively be the associated Galois representation and automorphic representation. Let $f$ be nearly ordinary at every prime $\mathfrak{p}$ in $F, \mathfrak{p} \mid p$. Then for any such $\mathfrak{p}$ dividing $p$, the Frobenius $\operatorname{Fr}_{\mathfrak{p}}$ acts on the 1 dimensional subspace $V_{f, \mathfrak{p}}^{-}$with the eigenvalue $\alpha_{f, \mathfrak{p}}$ (say).

Definition 2.1. Let $f$ be as above. Define $f$ to be exceptional if $\left|\alpha_{f, \mathfrak{p}}\right| \mathbb{C}=1$ for some $\mathfrak{p} \mid p$.

Remark 2.2. From the local Langlands correspondence for Hilbert modular forms due to Carayol [Ca] and generalized Ramanujan Conjecture, which is known for Hilbert modular form due to Blassias [B1]; it follows that the condition of $f$ being exceptional i.e. $\left|\alpha_{f, \mathfrak{p}}\right| \mathbb{C}=1$ for some $\mathfrak{p} \mid$ p happens if for some $\mathfrak{p} \mid p$, the $\mathfrak{p}$ component $\pi_{f, \mathfrak{p}}$ of the automorphic representation $\pi_{f}$ is Steinberg or its twist.

Theorem 2.3. Let $f$ be a Hilbert newform in $S_{k, w}^{n . o}\left(K_{1}(N) \cap K_{11}\left(p^{s}\right) ; \mathcal{O}\right)$ as above. Assume $f$ is not exceptional. Let $\rho$ be a character $\rho: \Gamma \longrightarrow O_{f}^{\times}$as above. Then the kernel and the cokernel of the map

$$
X\left(T_{f} \otimes T_{\rho} / F_{\text {cyc }}\right)_{\Gamma_{n}} \longrightarrow X\left(T_{f} \otimes T_{\rho} / F_{n}\right)
$$

are finite groups for all $n$ with their cardinality uniformly bounded independent of $n$.

Proof. Let $v_{n}$ be a prime of $F_{n}$ lying above $S$ and let $v_{c}$ be a prime of $F_{\text {cyc }}$ lying above it. Given such a prime $v_{n}$ we fix a prime $\bar{v}$ in $\overline{\mathbb{Q}}$ lying above it. Recall from $\S 1.2, G_{v_{n}}$ (resp. $G_{v_{c}}$ ) denotes the decomposition subgroup $\overline{\mathbb{Q}} / F_{n}$ (resp. $\overline{\mathbb{Q}} / F_{\text {cyc }}$ ) for the prime $\bar{v} / v_{n}$ (resp. $\left.\bar{v} / v_{\infty}\right)$. The corresponding inertia subgroups are $I_{v_{n}}$ and $I_{v_{c}}$ respectively. Similarly, $G_{v_{c} / v_{n}}$ (resp. $I_{v_{c} / v_{n}}$ ) denotes the decomposition subgroup (resp. inertia subgroup) of the Galois group of $F_{\text {cyc }} / F_{n}$ with respect to the primes $v_{\infty} / v_{n}$. Also the various Frobenius elements are given by $<\operatorname{Fr}_{\mathfrak{p}}>:=\frac{G_{\mathfrak{p}}}{I_{\mathfrak{p}}},<\operatorname{Fr}_{v_{n}}>:=\frac{G_{v_{n}}}{I_{v_{n}}}$ and

$$
\begin{aligned}
<\operatorname{Fr}_{v_{c} / v_{n}}>:=\frac{G_{v_{c} / v_{n}}}{I_{v_{c} / v_{n}}} \text {. Set } \\
J_{v_{n}}= \begin{cases}H^{1}\left(I_{v_{n}}, T_{\rho} \otimes A_{f}\right) & \text { if } v_{n} \mid S, v_{n} \nmid p \\
H^{1}\left(I_{v_{n}}, T_{\rho} \otimes\left(A_{f}\right)_{\mathfrak{p}}^{-}\right)^{G} v_{n} & \text { if } v_{n}|\mathfrak{p}| p,\end{cases} \\
J_{v_{c}}= \begin{cases}H^{1}\left(I_{v_{c}}, T_{\rho} \otimes A_{f}\right) & \text { if } v_{c} \in S, v_{c} \nmid p \\
H^{1}\left(I_{v_{c}}, T_{\rho} \otimes\left(A_{f}\right)_{\mathfrak{p}}^{-}\right)^{G v_{c}} & \text { if } v_{c}|\mathfrak{p}| p,\end{cases}
\end{aligned}
$$


We study the commutative diagram

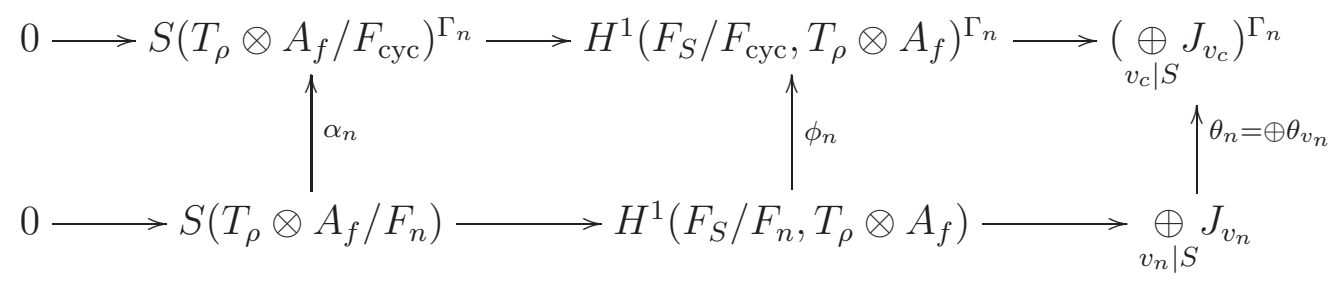

First, we prove that $\operatorname{ker}\left(\alpha_{n}\right)$ is finite for all $n$. We will show $\operatorname{ker}\left(\phi_{n}\right)$ is finite for all $n$. Note that $\operatorname{ker}\left(\phi_{n}\right) \cong H^{1}\left(\Gamma_{n},\left(T_{\rho} \otimes A_{f}\right)^{G_{F_{\mathrm{cyc}}}}\right)$. As $\Gamma_{n}$ is topologically cyclic and $T_{\rho} \otimes A_{f}$ is a cofinitely generated $\mathbb{Z}_{p}$ module is follows that $\operatorname{ker}\left(\phi_{n}\right)$ is finite if and only if $H^{0}\left(\Gamma_{n},\left(T_{\rho} \otimes A_{f}\right)^{G_{F_{\mathrm{cyc}}}}\right)$ is finite. Also, to show $\left(T_{\rho} \otimes A_{f}\right)^{G_{F_{n}}}$ is finite, it suffices to show $V_{f}(\rho)^{G_{F_{n}}}=0$. If $V_{f}(\rho)^{G_{F_{n}}} \neq 0$, then $V_{f}(\rho)$ contains a trivial $G_{F_{n}}$ sub-representation. In that case, we choose a place $\lambda \nmid N p$ and then restrict $G_{F}$ representation to $\operatorname{Fr}_{\lambda}$. Then considering the eigenvalues of $\operatorname{Fr}_{\lambda}$, we immediately get a contradiction by Theorem 1.5(1)(a), Theorem 1.5(1)(b).

As $H^{0}\left(F_{n}, V_{f}(\rho)\right)=0$ for every $n$ and $\operatorname{ker}\left(\alpha_{n}\right)$ is finite for all $n$, we deduce from [Oc2, Theorem 3.5(i)] that $\operatorname{ker}\left(\alpha_{n}\right)$ is uniformly bounded independent of $n$.

For coker $\left(\alpha_{n}\right)$, first note that $\operatorname{coker}\left(\phi_{n}\right) \subset H^{2}\left(\Gamma_{n},\left(T_{\rho} \otimes A_{f}\right)^{G_{F_{\mathrm{cyc}}}}\right)=0$ as $p$ cohomological dimension of $\Gamma_{n}=1$ for any $n$. Using the Snake lemma, it suffices to show that the kernel of $\theta_{n}$ are finite and uniformly bounded independent of $n$. Now, there are only finite number of primes in $F_{\text {cyc }}$ lying above a given prime in any $F_{n}$. Hence it is enough to prove $\operatorname{ker}\left(\theta_{v_{n}}\right)$ is finite for each $v_{n} \mid S$. Now for a prime $v_{n} \mid S$ such that $v_{n} \nmid p$, we have

$$
\operatorname{ker}\left(H^{1}\left(I_{v_{n}}, T_{\rho} \otimes A_{f}\right) \longrightarrow H^{1}\left(I_{v_{c}}, T_{\rho} \otimes A_{f}\right)\right) \cong H^{1}\left(I_{v_{n}} / I_{v_{c}}, T_{\rho} \otimes A_{f}^{I_{v_{c}}}\right) .
$$

The last isomorphism follows from the inflation-restriction sequence of Galois cohomology. We know that the cyclotomic $\mathbb{Z}_{p}$ extension of any number field is unramified outside primes above $p$. Thus $I_{v_{n}} / I_{v_{c}} \cong I_{v_{c} / v_{n}}=0$ whenever $v_{n} \nmid p$. Using (16) in diagram (15), it is immediate that $\operatorname{ker}\left(\theta_{v_{n}}\right)$ vanishes for $v_{n} \nmid p$.

To consider $\operatorname{ker}\left(\theta_{v_{n}}\right)$ for primes $v_{n}|\mathfrak{p}| p$, we study

$$
\begin{array}{r}
\operatorname{ker}\left(H ^ { 1 } \left(I_{v_{n}}, T_{\rho} \otimes\right.\right. \\
\left.\left.\left(A_{f}\right)_{\mathfrak{p}}^{-}\right)^{G_{v_{n}}} \longrightarrow H^{1}\left(I_{v_{c}}, T_{\rho} \otimes\left(A_{f}\right)_{\mathfrak{p}}^{-}\right)^{G_{v_{c}}}\right) \\
\cong H^{1}\left(I_{v_{c} / v_{n}},\left(T_{\rho} \otimes\left(A_{f}\right)_{\mathfrak{p}}^{-}\right)^{G_{v_{c}}}\right)^{<\operatorname{Fr}_{v_{c} / v_{n}}>}
\end{array}
$$

As, $\rho$ acts trivially on $G_{v_{c}}$,

$$
H^{1}\left(I_{v_{c} / v_{n}},\left(T_{\rho} \otimes\left(A_{f}\right)_{\mathfrak{p}}^{-}\right)^{G_{v_{c}}}\right) \cong H^{1}\left(I_{v_{c} / v_{n}}, T_{\rho} \otimes\left(\left(A_{f}\right)_{\mathfrak{p}}^{-}\right)^{G_{v_{c}}}\right)
$$

Now $F_{\text {cyc }} / F$ is totally ramified at $\mathfrak{p}$ for all $\mathfrak{p} \mid p$. Hence, $I_{v_{c} / v_{n}} \cong \mathbb{Z}_{p}$ for every $v_{n}$ lying above $\mathfrak{p} \mid p$. Note that, as an abstract group $T_{\rho} \cong O_{f},\left(\left(A_{f}\right)_{\mathfrak{p}}^{-}\right)^{\vee} \cong O_{f}$. Hence $H^{1}\left(I_{v_{c} / v_{n}}, T_{\rho} \otimes\left(A_{f}\right)_{\mathfrak{p}}^{-G_{v_{c}}}\right)=0$ unless $I_{v_{c} / v_{n}}$ acts trivially on $T_{\rho} \otimes\left(\left(A_{f}\right)_{\mathfrak{p}}^{-}\right)^{G_{v_{c}}}$.

Thus it suffices to consider the case where $I_{v_{c} / v_{n}}$ is acting trivially on $T_{\rho} \otimes$ $\left(\left(A_{f}\right)_{\mathfrak{p}}^{-}\right)^{G_{v_{c}}}$, as otherwise kernel in (17) is 0 . Then

$$
H^{1}\left(I_{v_{c} / v_{n}}, T_{\rho} \otimes\left(A_{f}\right)_{\mathfrak{p}}^{-G_{v_{c}}}\right) \cong \underset{14}{\operatorname{Hom}}\left(I_{v_{c} / v_{n}},\left(T_{\rho} \otimes\left(A_{f}\right)_{\mathfrak{p}}^{-}\right)^{G_{v_{c}}}\right)
$$


As $F_{\text {cyc }} / F$ is abelian, the action of the Frobenius $<\operatorname{Fr}_{v_{c} / v_{n}}>$ on $I_{v_{c} / v_{n}}$ (via lifting and conjugation) is trivial. Hence, the module in (17) is isomorphic to

$$
\begin{aligned}
& \operatorname{Hom}_{<\mathrm{Fr}_{v_{c} / v_{n}}>}\left(I_{v_{c} / v_{n}},\left(T_{\rho} \otimes\left(A_{f}\right)_{\mathfrak{p}}^{-}\right)^{G_{v_{c}}}\right) \\
& \cong \operatorname{Hom}\left(I_{v_{c} / v_{n}},\left(T_{\rho} \otimes\left(A_{f}\right)_{\mathfrak{p}}^{-}\right)^{G_{v_{n}}}\right) \\
& \cong \operatorname{Hom}\left(I_{v_{c} / v_{n}},\left(T_{\rho} \otimes\left(A_{f}\right)_{\mathfrak{p}}^{-}\right)^{\frac{G_{v_{n}}}{I v_{n}}}\right)
\end{aligned}
$$

We claim that $\left(T_{\rho} \otimes\left(A_{f}\right)_{\mathfrak{p}}^{-}\right)^{<\operatorname{Fr}_{v_{n}}>}$ is finite. On the one dimensional vector space corresponding to $\left(A_{f}\right)_{\mathfrak{p}}^{-}, \mathrm{Fr}_{\mathfrak{p}}$ acts by multiplication by $\alpha_{\mathfrak{p}}(f)$. By our assumption in this theorem that $f$ is not exceptional and remark 2.2, it follows that the eigenvalue of $\operatorname{Fr}_{\mathfrak{p}}$ acting on the 1 dimensional line corresponding to $\left(A_{f}\right)_{\mathfrak{p}}^{-}$is not a root of unity. On the other hand, $\rho(g)=1$ for any $g \in G_{F_{\mathrm{cyc}}}$ and $F_{\text {cyc }} / F$ is totally ramified at any prime in $F$ lying above $p$. Hence the eigenvalue corresponding to the action of $\mathrm{Fr}_{\mathfrak{p}}$ on $T_{\rho}$ is a root of unity. Combining these facts, we deduce that the eigenvalue of $\operatorname{Fr}_{\mathfrak{p}}$ on the line corresponding to $T_{\rho} \otimes\left(A_{f}\right)_{\mathfrak{p}}^{-}$is not a root of unity. Hence $\operatorname{Fr}_{v_{n}}$ acts non-trivially on $T_{\rho} \otimes\left(A_{f}\right)_{\mathfrak{p}}^{-}$for any $n$ and any $\rho$ as before. Hence $\left(T_{\rho} \otimes\left(A_{f}\right)_{\mathfrak{p}}^{-}\right)^{<\operatorname{Fr}_{v_{n}}>}$ is indeed finite. Also as $\left\langle\operatorname{Fr}_{v_{n}}\right\rangle \cong\left\langle\mathrm{Fr}_{v_{c}}\right\rangle$ for $n \gg 0$, we deduce that the size of $\left(T_{\rho} \otimes\left(A_{f}\right)_{\mathfrak{p}}^{-}\right)^{<\operatorname{Fr}_{v_{n}}>}$ is independent of $n$ for large enough $n$. As $I_{v_{c} / v_{n}} \cong \mathbb{Z}_{p}$ for every $n$; for any $\rho$ and every $n \geq 0$, the module in (17), given by $\operatorname{Hom}\left(I_{v_{c} / v_{n}},\left(T_{\rho} \otimes\right.\right.$ $\left.\left(A_{f}\right)_{\mathfrak{p}}^{-}\right)^{<\operatorname{Fr}_{v_{n}}>}$ ), is also finite with its cardinality bounded independent of $n$. Hence same is true for $\operatorname{ker}\left(\theta_{v_{n}}\right)$ for $v_{n} \mid \mathfrak{p}$. This completes the proof.

Let $O$ be the ring of integers of a finite extension of $\mathbb{Q}_{p}$. Take $M$ to be a finitely generated $\Lambda$ module, where $\Lambda=O[[\Gamma]]$. Let us denote $\operatorname{Ext}_{\Lambda}{ }^{1}(M, \Lambda)$ by $a_{\Lambda}^{1}(M)$.

Lemma 2.4. Let $M$ be a finitely generated torsion $\Lambda=O[[\Gamma]]$ module such that $M_{\Gamma_{n}}$ is finite for each $n$. Then

$$
a_{\Lambda}^{1}(M) \cong{\underset{n}{n}}_{\lim _{n}}\left(M^{\iota \vee}\right)^{\Gamma_{n}}
$$

Proof. See [Pe, §1.3, page 733].

Lemma 2.5. Let $M$ be a finitely generated torsion $\Lambda=O[[\Gamma]]$ module. Then $C h_{\Lambda}(M)=C h_{\Lambda}\left(a_{\Lambda}^{1}(M)\right)$, considered as ideals in $O[[\Gamma]]$.

Proof. See [J-P, Lemma 3.5]).

Lemma 2.6. Let $M$ be a finitely generated torsion $\Lambda=O[[\Gamma]]$ module. Then there exists a character $\rho: \Gamma=\operatorname{Gal}\left(F_{\text {cyc }} / F\right) \longrightarrow$ Aut $(O)$ such that $(M(\rho))_{\Gamma_{n}}$ is finite for every $n$, where $M(\rho)$ is as defined in section 1.2.

Proof. This is well known, for example see [Pe, §2.6, Page 740].

Lemma 2.7. For any cuspidal Hilbert newform $f$, the dual Selmer groups $X\left(T_{f} / F_{\text {cyc }}\right)$ and $X\left(T_{f}^{*} / F_{\text {cyc }}\right)$ are finitely generated $O_{f}[[\Gamma]]$ modules.

Proof. The proof is similar to the one in elliptic modular form case (see for example [J-P, Lemma 3.7]).

Throughout the rest of the article we make this assumption - 
Hypothesis 2.8. $($ Tor $)=\left(\right.$ Tor $\left._{f}\right)$ : For any cuspidal Hilbert newform $f, X\left(T_{f} / F_{\text {cyc }}\right)$ is finitely generated torsion $O_{f}[[\Gamma]]$ module.

Corollary 2.9. It follows from remark 1.20 that by hypothesis (Tor), we have for any cuspidal Hilbert newform $f, X\left(T_{f}^{*} / F_{\text {cyc }}\right)$ is also torsion over $O_{f}[[\Gamma]]$.

Theorem 2.10. Let the notation be as before. Let $f \in S_{k, w}^{n . o}\left(K_{1}(N) \cap K_{11}\left(p^{s}\right) ; \mathcal{O}\right)$ be a Hilbert newform nearly ordinary at $\mathfrak{p} \mid p$, which is not exceptional (as defined in theorem 2.3). Assume (Tor) holds. Then the functional equation holds for $X\left(T_{f} / F_{\text {cyc }}\right)$ i.e. we have the equality of ideals in $O_{f}[[\Gamma]]$,

$$
C h_{O_{f}[[\Gamma]]}\left(X\left(T_{f} / F_{\text {cyc }}\right)\right)=C h_{O_{f}[[\Gamma]]}\left(X\left(T_{f}^{*} / F_{\text {cyc }}\right)^{\iota}\right) .
$$

Proof. By the assumption (Tor) and corollary 2.9 , both $X\left(T_{f} / F_{\text {cyc }}\right)$ and $X\left(T_{f}^{*} / F_{\text {cyc }}\right)$ are torsion over $O_{f}[[\Gamma]]$. Thus we can find a $\rho$ by lemma 2.6 such that $X\left(T_{f} \otimes T_{\rho} / F_{n}\right)$ and $X\left(T_{f}^{*} \otimes T_{\rho^{-1}} / F_{n}\right)$ are both finite groups for every $n$. Then from the generalized Cassels-Tate pairing of Flach (see [Pe, 3.1.1]), we obtain that $S\left(T_{\rho^{-1}} \otimes A_{f}^{*} / F_{n}\right) \cong$ $X\left(T_{\rho} \otimes T_{f} / F_{n}\right)$ for every $n$. Hence we get

$$
X\left(T_{\rho} \otimes T_{f} / F_{\text {cyc }}\right) \cong \lim _{n} X\left(T_{\rho} \otimes T_{f} / F_{n}\right) \cong \underbrace{\lim _{n}}_{n} S\left(T_{\rho^{-1}} \otimes A_{f}^{*} / F_{n}\right) .
$$

By Theorem 2.3 and remark 1.20, we see that the kernel and the cokernel of the natural restriction map, given by $S\left(T_{\rho^{-1}} \otimes A_{f}^{*} / F_{n}\right) \stackrel{\alpha_{n}^{*}}{\longrightarrow} S\left(T_{\rho^{-1}} \otimes A_{f}^{*} / F_{\text {cyc }}\right)^{\Gamma_{n}}$, are finite groups and their size uniformly bounded independent of $n$. Thus we obtain from (18) that the induced map

$$
X\left(T_{\rho} \otimes T_{f} / F_{\text {cyc }}\right) \stackrel{\phi_{\rho}}{\longrightarrow} \underset{n}{\lim _{n}} S\left(T_{\rho^{-1}} \otimes A_{f}^{*} / F_{\text {cyc }}\right)^{\Gamma_{n}}
$$

is a $O_{f}[[\Gamma]]$ pseudo-isomorphism. We have

$\lim _{n} S\left(T_{\rho^{-1}} \otimes A_{f}^{*} / F_{\text {cyc }}\right)^{\Gamma_{n}}={\underset{l}{n}}_{\lim _{n}}\left(X\left(T_{\rho^{-1}} \otimes T_{f}^{*} / F_{\text {cyc }}\right)^{\vee}\right)^{\Gamma_{n}} \stackrel{\text { Lemma }}{\cong} a_{\Lambda}^{1.4}\left(X\left(T_{\rho^{-1}} \otimes T_{f}^{*} / F_{\text {cyc }}\right)^{\iota}\right)$.

Combining this with (19) we get an $O_{f}[[\Gamma]]$ module pseudo-isomorphism

$$
X\left(T_{f} \otimes T_{\rho} / F_{\mathrm{cyc}}\right) \stackrel{\theta_{\rho}}{\longrightarrow} a_{\Lambda}^{1}\left(X\left(T_{f}^{*} \otimes T_{\rho^{-1}} / F_{\mathrm{cyc}}\right)^{\iota}\right) .
$$

We recall from (14), $X\left(T_{f} \otimes T_{\rho} / F_{\text {cyc }}\right) \cong X\left(T_{f} / F_{\text {cyc }}\right) \otimes T_{\rho^{-1}}$. On the other hand, we have $a_{\Lambda}^{1}\left(X\left(T_{f}^{*} \otimes T_{\rho^{-1}} / F_{\text {cyc }}\right)^{\iota}\right) \cong a_{\Lambda}^{1}\left(X\left(T_{f}^{*} / F_{\text {cyc }}\right)^{\iota}\right) \otimes T_{\rho^{-1}}$ (see [Pe, Page 744, §3.2.13.2.2]). Thus tensoring (20) with $T_{\rho}$, we get a pseudo-isomorphism of $O_{f}[[\Gamma]]$ modules

$$
X\left(T_{f} / F_{\text {cyc }}\right) \longrightarrow a_{\Lambda}^{1}\left(X\left(T_{f}^{*} / F_{\text {cyc }}\right)^{\iota}\right)
$$

which is independent of $\rho$. Hence $C h_{O_{f}[[\Gamma]]}\left(X\left(T_{f} / F_{\text {cyc }}\right)\right)=C h_{O_{f}[[\Gamma]]}\left(a_{\Lambda}^{1}\left(X\left(T_{f}^{*} / F_{\text {cyc }}\right)^{\iota}\right)\right)$ as ideals in $O_{f}[[\Gamma]]$. By applying Lemma 2.5, we get that

$$
C h_{O_{f}[[\Gamma]]}\left(X\left(T_{f} / F_{\text {cyc }}\right)\right)=\underset{16}{C h_{O_{f}[[\Gamma]]}}\left(X\left(T_{f}^{*} / F_{\text {cyc }}\right)^{\iota}\right) .
$$




\section{FUNCTIONAL EQUATION FOR A NEARLY ORDINARY HIDA FAMILY}

We begin by proving a specialization result relating the 'big' Selmer group with the individual Selmer groups.

Theorem 3.1. Assume (Irr), (Dist). Let $\mathcal{R}$ be a branch of $\mathbf{H}_{\mathcal{N}, \mathcal{O}}$ and assume that $\mathcal{R}$ is a power series ring in many variable (i.e. we assume that $\mathcal{R} \cong O\left[\left[X_{1}, \cdots, X_{r}\right]\right]$, where $r=d+1+\delta_{F, p}$, here $\delta_{F, p}$ is the defect of Leopoldt's conjecture for $F$ at $p$, $d=[F: \mathbb{Q}]$, and $O$ is the ring of integer of some finite extension of $\left.\mathbb{Q}_{p}\right)$. Let $s_{\xi}^{\vee}$ be the natural specialization map

$$
\mathcal{X}\left(\mathcal{T}_{\mathcal{R}} / F_{\text {cyc }}\right) / P_{\xi} \mathcal{X}\left(\mathcal{T}_{\mathcal{R}} / F_{\text {cyc }}\right) \stackrel{s_{\xi}^{\vee}}{\longrightarrow} X\left(T_{f_{\xi}} / F_{\text {cyc }}\right)
$$

- Then the kernel and the cokernel of $s_{\xi}^{\vee}$ are finitely generated $\mathbb{Z}_{p}$ modules for every $\xi \in \mathfrak{X}(\mathcal{R})$ and

- There exists a non zero ideal $J$ in $\mathcal{R}$ such that for any $\xi \in \mathfrak{X}(\mathcal{R}) \backslash S_{J}$, the kernel and the cokernel of $s_{\xi}^{\vee}$ are finite, where the set $S_{J}$ is defined as $S_{J}:=\left\{\xi \in \mathfrak{X}(\mathcal{R}) \mid P_{\xi}\right.$ does not contain $\left.J\right\}$.

In particular, assuming (Tor), the equality

$$
C h_{O_{f_{\xi}}[[T]]}\left(\mathcal{X}\left(\mathcal{T}_{\mathcal{R}} / F_{\text {cyc }}\right) / P_{\xi} \mathcal{X}\left(\mathcal{T}_{\mathcal{R}} / F_{\text {cyc }}\right)\right)=C h_{\left.O_{f_{\xi}}[T]\right]}\left(X\left(T_{f_{\xi}} / F_{\text {cyc }}\right)\right)
$$

holds for all $\xi \in \mathfrak{X}(\mathcal{R}) \backslash S_{J}$.

Proof. For a finitely generated $\mathcal{R}$ module $M$, we define $M^{\ddagger}:=\operatorname{Hom}_{\mathcal{R}}(M, \mathcal{R})$. For convenience of notation, let $\mathcal{T}=\mathcal{T}_{\mathcal{R}}$ and $\mathcal{A}=\mathcal{A}_{\mathcal{R}}$. As before, $v_{c}$ will denote a prime in $F_{\text {cyc }}$ lying above $S$ and $I_{c}$ denotes the inertia subgroup of $\overline{\mathbb{Q}} / F_{\text {cyc }}$ with respect to the primes $\bar{v} / v_{c}$. We have the commutative diagram with the natural maps

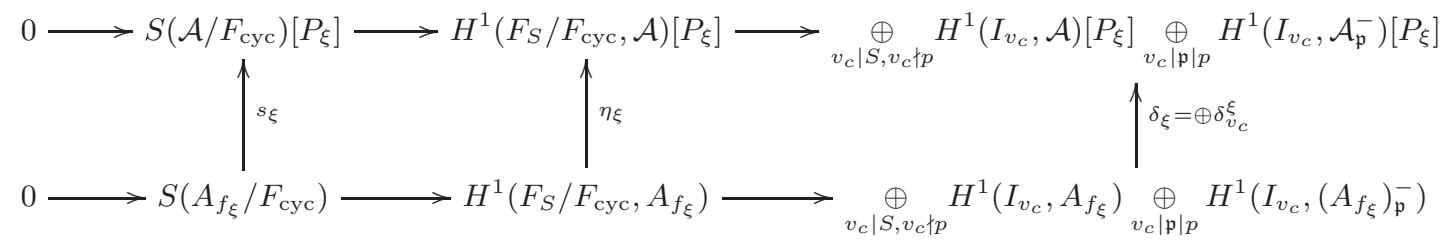

Recall, $A_{f_{\xi}} \cong \mathcal{A}\left[P_{\xi}\right]$ and $\left(A_{f_{\xi}}\right)_{\mathfrak{p}}^{-} \cong \mathcal{A}_{\mathfrak{p}}^{-}\left[P_{\xi}\right]$. Since we assume that the residual representation is absolutely irreducible, we have (by [Gr2, Remark 3.4.1])

$$
H^{1}\left(F_{S} / F_{\text {cyc }}, \mathcal{A}\left[P_{\xi}\right]\right) \stackrel{\eta_{\xi}}{\longrightarrow} H^{1}\left(F_{S} / F_{\text {cyc }}, \mathcal{A}\right)\left[P_{\xi}\right]
$$

is an isomorphism. By Snake lemma, we get that $\operatorname{ker}\left(s_{\xi}\right)$ is trivial for every $\xi$. Thus $\operatorname{coker}\left(s_{\xi}^{\vee}\right)=0$.

Next we want to prove $\operatorname{ker}\left(s_{\xi}^{\vee}\right)$ is finitely generated $\mathbb{Z}_{p}$ module for every $\xi$. As there are only finitely many primes in $F_{\text {cyc }}$ lying over a given prime in $F$, it is enough to show that $\operatorname{ker}\left(\delta_{v_{c}}^{\xi}\right)^{\vee}$ is finitely generated $\mathbb{Z}_{p}$ module for all $v_{c} \mid S$. By our assumption, $\mathcal{R} \cong O\left[\left[X_{1}, \cdots, X_{r}\right]\right]$, where $r=[F: \mathbb{Q}]+1+\delta_{F, p}$, where $\delta_{F, p}$ is the defect of Leopoldt's conjecture for $F$ at $p$ and $O$ is some finite extension of $\mathbb{Z}_{p}$. Since $\mathcal{R}$ is regular local and $P_{\xi}$ is a prime ideal of height $r$, we have $P_{\xi}=\left(x_{1}, \cdots, x_{r}\right)$, here $x_{1}, \cdots, x_{r}$ is a regular sequence of prime elements of $\mathcal{R}$. Define $P_{0}=(0)$ and 
$P_{i}=\left(x_{1}, \cdots, x_{i}\right)$, for $1 \leq i \leq r$. Then $P_{i}$ is a prime ideal and $\mathcal{A}\left[P_{i}\right]$ is divisible as $\mathcal{R} / P_{i}$ module. Notice that $\mathcal{A}\left[P_{i}\right]=\left(\mathcal{A}\left[P_{i-1}\right]\right)\left[x_{i}\right]$, and multiplication by $x_{i}$ is surjective on $\mathcal{A}\left[P_{i-1}\right]$. We get a induced map of $\mathcal{R} / P_{i}$ modules

$$
0 \rightarrow \mathcal{A}^{I_{v_{c}}}\left[P_{i-1}\right] / x_{i} \mathcal{A}^{I_{v_{c}}}\left[P_{i-1}\right] \rightarrow H^{1}\left(I_{v_{c}}, \mathcal{A}\left[P_{i}\right]\right) \rightarrow H^{1}\left(I_{v_{\infty}}, \mathcal{A}\left[P_{i-1}\right]\right)\left[x_{i}\right] \rightarrow 0 .
$$

Thus we can obtain kernel of $\delta_{v_{c}}^{\xi}$ via successive extensions. Let $\operatorname{Ker}_{1}=\mathcal{A}^{I_{v_{c}}} / x_{1} \mathcal{A}^{I_{v_{c}}}$, and let $\operatorname{Ker}_{i}$ denotes the kernel of the map $H^{1}\left(I_{v_{c}}, \mathcal{A}\left[P_{i}\right]\right) \rightarrow H^{1}\left(I_{v_{c}}, \mathcal{A}\right)\left[P_{i}\right]$, then $K_{e r}$ is an extension of the form,

$$
0 \rightarrow \mathcal{A}^{I_{v_{c}}}\left[P_{i-1}\right] / x_{i} \mathcal{A}^{I_{v_{c}}}\left[P_{i-1}\right] \rightarrow \operatorname{Ker}_{i} \rightarrow \operatorname{Ker}_{(i-1)}\left[x_{i}\right] \rightarrow 0 .
$$

Then in this notation $\operatorname{ker}\left(\delta_{v_{c}}^{\xi}\right)=\operatorname{Ker}_{r}$.

First, we show that $\left(\operatorname{Ker}_{r}\right)^{\vee}$ is finitely generated $\mathbb{Z}_{p}$ module for every $\xi$. We also denote $I_{v_{c}}$ by $G$ for the rest of this proof. Taking Pontryagin dual, we get from (24), for each $i$,

$$
0 \rightarrow \frac{\operatorname{Ker}_{i-1}^{\vee}}{x_{i} \operatorname{Ker}_{i-1}^{\vee}} \rightarrow \operatorname{Ker}_{i}^{\vee} \rightarrow \frac{\mathcal{T}_{G}^{\ddagger}}{P_{i-1} \mathcal{T}_{G}^{\ddagger}}\left[x_{i}\right] \rightarrow 0 .
$$

Now for $i=1, \operatorname{Ker}_{1}^{\vee} \cong \mathcal{T}_{G}^{\ddagger}\left[x_{1}\right]$ is a finitely generated $\mathcal{R} / x_{1}$ module. By induction, assume that for $i=1,2, \cdots, r-1, \operatorname{Ker}_{i}^{\vee}$ is a finitely generated $\mathcal{R} /\left(x_{1}, \cdots, x_{i}\right)$ module. Also, $\mathcal{T}_{G}^{\ddagger}$ being a finitely generated $\mathcal{R}$ module, it is immediate that $\mathcal{T}_{G}^{\ddagger} / P_{r-1} \mathcal{T}_{G}^{\ddagger}$ is a finitely generated $\mathcal{R} / P_{r-1}$ module. Consequently, $\mathcal{T}_{G}^{\ddagger} / P_{r-1} \mathcal{T}_{G}^{\ddagger}\left[x_{r}\right]$ is a finitely generated $\mathcal{R} / P_{r}$ module. Also by induction hypothesis, $\operatorname{Ker}_{r-1}^{\vee} / x_{r}$ is a finitely generated $\mathcal{R} /\left(P_{r-1}, x_{r}\right) \cong R / P_{r}$ module. Hence, we deduce from (25), that $\operatorname{Ker}_{r}^{\vee}$ is a finitely generated $\mathcal{R} / P_{r}$ module. Recall, in this notation, $P_{r}=\left(x_{1}, \cdots, x_{r}\right)=P_{\xi}$ and hence $\mathcal{R} / P_{\xi} \cong O$, a finite extension of $\mathbb{Z}_{p}$. This finishes the proof of the first assertion of the theorem.

For the second assertion of the theorem, we have to show that there exists a non zero ideal $J$ in $\mathcal{R}$ such that for any $\xi \in \mathfrak{X}(\mathcal{R})$ such that $\operatorname{ker}(\xi)=P_{\xi} \nsupseteq J$, the rank

$$
\operatorname{rk}_{\mathcal{R} /\left(x_{1}, \cdots, x_{r}\right)} \operatorname{Ker}_{r}^{\vee}=0 .
$$

By [Gr2, Theorem 2.1], corresponding to the $\mathcal{R}$ module $\mathcal{T}_{G}^{\ddagger}$ there exists an ideal $J \neq 0$ such that the following two equations hold

$$
\begin{gathered}
\operatorname{rk}_{\mathcal{R} / P} \mathcal{T}_{G}^{\ddagger}[P]=0 \text { for any prime ideal } \mathrm{P} \nsupseteq J \\
\operatorname{rk}_{\mathcal{R} / P} \frac{\mathcal{T}_{G}^{\ddagger}}{P \mathcal{T}_{G}^{\ddagger}}=\mathrm{rk}_{\mathcal{R}} \mathcal{T}_{G}^{\ddagger} \text { for any prime ideal } P \nsupseteq J
\end{gathered}
$$

We will go on to show that this $J$ will work for us. Let $P_{\xi}=\left(x_{1}, \cdots, x_{r}\right) \nsupseteq J$. Then as $\left(x_{1}\right) \nsupseteq J$, we have

$$
\operatorname{rk}_{\mathcal{R} / x_{1}} \mathcal{T}_{G}^{\ddagger}\left[x_{1}\right]=0 .
$$

But $\operatorname{Ker}_{1}^{\vee} \cong \mathcal{T}_{G}^{\ddagger}\left[x_{1}\right]$. Thus we get that

$$
\operatorname{rk}_{\mathcal{R} / x_{1}} \operatorname{Ker}_{1}^{\vee}=0 .
$$

Let us assume for $i=1,2, \cdots, r-1$,

$$
\operatorname{rk}_{\mathcal{R} /\left(x_{1}, \cdots, x_{i}\right)} \operatorname{Ker}_{i}^{\vee}=0 .
$$


Notice that

$$
\begin{aligned}
\operatorname{rk}_{\mathcal{R} /\left(x_{1}, \cdots, x_{r}\right)} \frac{\mathcal{T}_{G}^{\ddagger}}{P_{r-1} T_{G}^{\ddagger}}\left[x_{r}\right] & =\operatorname{rk}_{\mathcal{R} /\left(x_{1}, \cdots, x_{r}\right)} \frac{\mathcal{T}_{G}^{\ddagger}}{\left(P_{r-1}, x_{r}\right) T_{G}^{\ddagger}}-\operatorname{rk}_{\mathcal{R} /\left(x_{1}, \cdots, x_{r-1}\right)} \frac{\mathcal{T}_{G}^{\ddagger}}{P_{r-1} T_{G}^{\ddagger}} \\
& =\operatorname{rk}_{\mathcal{R} /\left(x_{1}, \cdots, x_{r}\right)} \frac{\mathcal{T}_{G}^{\ddagger}}{P_{r} T_{G}^{\ddagger}}-\operatorname{rk}_{\mathcal{R} /\left(x_{1}, \cdots, x_{r-1}\right)} \frac{\mathcal{T}_{G}^{\ddagger}}{P_{r-1} T_{G}^{\ddagger}} .
\end{aligned}
$$

By our assumption that $P_{r} \nsupseteq J$, we deduce from (27) that

$$
\operatorname{rk}_{\mathcal{R} / P_{r}} \frac{\mathcal{T}_{G}^{\ddagger}}{P_{r} \mathcal{T}_{G}^{\ddagger}}=\operatorname{rk}_{\mathcal{R} / P_{r-1}} \frac{\mathcal{T}_{G}^{\ddagger}}{P_{r-1} \mathcal{T}_{G}^{\ddagger}}=\operatorname{rk}_{\mathcal{R}} \mathcal{T}_{G}^{\ddagger} .
$$

Hence we deduce from (30) that $\mathrm{rk}_{\mathcal{R} / P_{r}} \frac{\mathcal{T}_{G}^{\ddagger}}{P_{r-1} T_{G}^{\ddagger}}\left[x_{r}\right]=0$.

Thus using (25), to finish the proof of the theorem, it suffices to show that

$$
\mathrm{rk}_{\mathcal{R} / P_{r}} \operatorname{Ker}_{r-1}^{\vee} / x_{r}=0 .
$$

Now

$$
\begin{aligned}
\operatorname{rk}_{\mathcal{R} /\left(x_{1}, \cdots, x_{r}\right)} \frac{\operatorname{Ker}_{r-1}^{\vee}}{x_{r} \operatorname{Ker}_{r-1}^{\vee}} & =\operatorname{rk}_{\mathcal{R} /\left(x_{1}, \cdots, x_{r-1}\right)} \operatorname{Ker}_{r-1}^{\vee}+\operatorname{rk}_{\mathcal{R} /\left(x_{1}, \cdots, x_{r}\right)} \operatorname{Ker}_{r-1}^{\vee}\left[x_{r}\right] \\
& =\operatorname{rk}_{\mathcal{R} /\left(x_{1}, \cdots, x_{r}\right)} \operatorname{Ker}_{r-1}^{\vee}\left[x_{r}\right],
\end{aligned}
$$

where the last equality follows from (29). Thus to prove (31), we are further reduced to showing

$$
\operatorname{rk}_{\mathcal{R} /\left(x_{1}, \cdots, x_{r}\right)} \operatorname{Ker}_{r-1}^{\vee}\left[x_{r}\right]=0
$$

Now we have the exact sequence,

$$
0 \rightarrow \frac{\operatorname{Ker}_{r-2}^{\vee}}{x_{r-1} \operatorname{Ker}_{r-2}^{\vee}}\left[x_{r}\right] \rightarrow \operatorname{Ker}_{r-1}^{\vee}\left[x_{r}\right] \rightarrow \frac{\mathcal{T}_{G}^{\ddagger}}{P_{r-2} \mathcal{T}_{G}^{\ddagger}}\left[\left(x_{r-1}, x_{r}\right)\right]
$$

Proceeding similarly as in (30), we deduce that

$$
\begin{aligned}
\operatorname{rk}_{\mathcal{R} / P_{r}} \frac{\mathcal{T}_{G}^{\ddagger}}{P_{r-2} \mathcal{T}_{G}^{\ddagger}}\left[\left(x_{r-1}, x_{r}\right)\right] & =\operatorname{rk}_{\mathcal{R} / P_{r}} \frac{\mathcal{T}_{G}^{\ddagger}}{P_{r} T_{G}^{\ddagger}}-\operatorname{rk}_{\mathcal{R} / P_{r-2}} \frac{\mathcal{T}_{G}^{\ddagger}}{P_{r-2} T_{G}^{\ddagger}} \\
& =0 .
\end{aligned}
$$

Thus

$$
\begin{aligned}
\operatorname{rk}_{\mathcal{R} / P_{r}} \operatorname{Ker}_{r-1}^{\vee}\left[x_{r}\right] & =\operatorname{rk}_{\mathcal{R} / P_{r}} \frac{\operatorname{Ker}_{r-2}^{\vee}}{x_{r-1} \operatorname{Ker}_{r-2}^{\vee}}\left[x_{r}\right] \\
& =\operatorname{rk}_{\mathcal{R} / P_{r}} \frac{\operatorname{Ker}_{r-2}^{\vee}}{\left(x_{r-1}, x_{r}\right) \operatorname{Ker}_{r-2}^{\vee}}-\operatorname{rk}_{\mathcal{R} / P_{r-1}} \frac{\operatorname{Ker}_{r-2}^{\vee}}{x_{r-1} \operatorname{Ker}_{r-2}^{\vee}} .
\end{aligned}
$$

Now, note that

$$
\begin{aligned}
\operatorname{rk}_{\mathcal{R} / P_{r}} \frac{\operatorname{Ker}_{r-2}^{\vee}}{\left(x_{r-1}, x_{r}\right) \operatorname{Ker}_{r-2}^{\vee}} & =\operatorname{rk}_{\mathcal{R} / P_{r-2}} \operatorname{Ker}_{r-2}^{\vee}+\operatorname{rk}_{\mathcal{R} / P_{r}} \operatorname{Ker}_{r-2}^{\vee}\left[\left(x_{r-1}, x_{r}\right)\right] \\
& =\operatorname{rk}_{\mathcal{R} / P_{r}} \operatorname{Ker}_{r-2}^{\vee}\left[\left(x_{r-1}, x_{r}\right)\right] .
\end{aligned}
$$


Here the last equality follows from the induction hypothesis in (29). Using this in (36), we deduce that

$$
\operatorname{rk}_{\mathcal{R} / P_{r}} \operatorname{Ker}_{r-1}^{\vee}\left[x_{r}\right]=\operatorname{rk}_{\mathcal{R} / P_{r}} \operatorname{Ker}_{r-2}^{\vee}\left[\left(x_{r-1}, x_{r}\right)\right]-\operatorname{rk}_{\mathcal{R} / P_{r-1}} \frac{\operatorname{Ker}_{r-2}^{\vee}}{x_{r-1} \operatorname{Ker}_{r-2}^{\vee}}
$$

Recall, from (25), for $i=r-1$, we have the exact sequence

$$
0 \rightarrow \frac{\operatorname{Ker}_{r-2}^{\vee}}{x_{r-1} \operatorname{Ker}_{r-2}^{\vee}} \rightarrow \operatorname{Ker}_{r-1}^{\vee} \rightarrow \frac{\mathcal{T}_{G}^{\ddagger}}{P_{r-2} \mathcal{T}_{G}^{\ddagger}}\left[x_{r-1}\right] \rightarrow 0 .
$$

Using induction hypothesis (29) in (39), we deduce that

$$
\operatorname{rk}_{\mathcal{R} / P_{r-1}} \frac{\operatorname{Ker}_{r-2}^{\vee}}{x_{r-1} \operatorname{Ker}_{r-2}^{\vee}}=0 .
$$

Hence we obtain from (38) that

$$
\operatorname{rk}_{\mathcal{R} / P_{r}} \operatorname{Ker}_{r-1}^{\vee}\left[x_{r}\right]=\operatorname{rk}_{\mathcal{R} / P_{r}} \operatorname{Ker}_{r-2}^{\vee}\left[\left(x_{r-1}, x_{r}\right)\right]
$$

Proceeding in a similar way, we deduce that

$$
\begin{aligned}
\operatorname{rk}_{\mathcal{R} / P_{r}} \operatorname{Ker}_{r}^{\vee} & =\operatorname{rk}_{\mathcal{R} / P_{r}} \operatorname{Ker}_{r-1}^{\vee}\left[x_{r}\right] \\
& =\operatorname{rk}_{\mathcal{R} / P_{r}} \operatorname{Ker}_{r-2}^{\vee}\left[\left(x_{r-1}, x_{r}\right)\right] \\
& =\operatorname{rk}_{\mathcal{R} / P_{r}} \operatorname{Ker}_{1}^{\vee}\left[\left(x_{2}, \cdots, x_{r}\right)\right] \\
& =\operatorname{rk}_{\mathcal{R} / P_{r}} \mathcal{T}_{G}^{\ddagger}\left[x_{1}\right]\left[\left(x_{2}, \cdots, x_{r}\right)\right] \\
& =\operatorname{rk}_{\mathcal{R} / P_{r}} \mathcal{T}_{G}^{\ddagger}\left[\left(x_{1}, x_{2}, \cdots, x_{r}\right)\right] \\
& =\operatorname{rk}_{\mathcal{R} / P_{r}} \mathcal{T}_{G}^{\ddagger}\left[\left(x_{1}, x_{2}, \cdots, x_{r}\right)\right] \\
& =\operatorname{rk}_{\mathcal{R} / P_{r}} \mathcal{T}_{G}^{\ddagger}\left[P_{r}\right]=0 .
\end{aligned}
$$

Here the last equality follows from our hypothesis that $P_{r}=P_{\xi} \nsupseteq J$. This finishes the proof of the second assertion of the theorem.

Remark 3.2. Let the assumptions be as in Theorem 3.1. Then proceeding as in the proof of Theorem 3.1, we can deduce the corresponding theorem for $\mathcal{T}_{\mathcal{R}}^{*}$ i.e. there exists a non zero ideal $J^{*}$ in $\mathcal{R}$ such that for any $\xi \in \mathfrak{X}(\mathcal{R}) \backslash S_{J^{*}}$, the kernel and the cokernel of $s_{\xi}^{* \vee}$ are finite, where $S_{J^{*}}:=\left\{\xi \in \mathfrak{X}(\mathcal{R}) \mid P_{\xi}\right.$ does not contain $\left.J^{*}\right\}$. Consequently under the assumption (Tor),

$$
C h_{\left.O_{f_{\xi}}[\Gamma]\right]}\left(\mathcal{X}\left(\mathcal{T}_{\mathcal{R}}^{*} / F_{\text {cyc }}\right) / P_{\xi} \mathcal{X}\left(\mathcal{T}_{\mathcal{R}}^{*} / F_{\text {cyc }}\right)\right)=C h_{O_{f_{\xi}}[[\Gamma]]}\left(X\left(T_{f_{\xi}}^{*} / F_{\text {cyc }}\right)\right)
$$

for every $\xi \in \mathfrak{X}(\mathcal{R}) \backslash S_{J^{*}}$.

Remark 3.3. Under the assumption (Tor), by applying the involution $\iota$ we obtain,

$$
\begin{array}{r}
C h_{O_{f_{\xi}}[[\Gamma]]}\left(\mathcal{X}\left(\mathcal{T}_{\mathcal{R}}^{*} / F_{\text {cyc }}\right)^{\iota} / P_{\xi} \mathcal{X}\left(\mathcal{T}_{\mathcal{R}}^{*} / F_{\text {cyc }}\right)^{\iota}\right)=C h_{O_{f_{\xi}}[[\Gamma]]}\left(\left(\mathcal{X}\left(\mathcal{T}_{\mathcal{R}}^{*} / F_{\text {cyc }}\right) / P_{\xi} \mathcal{X}\left(\mathcal{T}_{\mathcal{R}}^{*} / F_{\text {cyc }}\right)\right)^{\iota}\right) \\
=C h_{\left.O_{f_{\xi}}[\Gamma]\right]}\left(X\left(T_{f_{\xi}}^{*} / F_{\text {cyc }}\right)^{\iota}\right) .
\end{array}
$$

for every $\xi \in \mathfrak{X}(\mathcal{R}) \backslash S_{J^{*}}$. 
Lemma 3.4. Assume (Irr), (Dist) and that $\mathcal{R}$ is a power series ring in many variables. Then $\mathcal{X}\left(\mathcal{T}_{\mathcal{R}} / F_{\text {cyc }}\right)$ and $\mathcal{X}\left(\mathcal{T}_{\mathcal{R}}^{*} / F_{\text {cyc }}\right)$ are finitely generated $\mathcal{R}[[\Gamma]]$ modules.

Proof. This result follows from Theorem 3.1, remark 3.2, Lemma 2.7 and topological Nakayama's Lemma [N-S-W, Corollary 5.2.18].

Corollary 3.5. Let the assumption be as in Theorem 3.1. Then under hypothesis (Tor), $\mathcal{X}\left(\mathcal{T}_{\mathcal{R}} / F_{\text {cyc }}\right)$ and $\mathcal{X}\left(\mathcal{T}_{\mathcal{R}}^{*} / F_{\text {cyc }}\right)$ are finitely generated torsion $\mathcal{R}[[\Gamma]]$ modules.

Proof. We will prove for $\mathcal{X}\left(\mathcal{T}_{\mathcal{R}} / F_{\text {cyc }}\right)$ and a similar argument works for $\mathcal{X}\left(\mathcal{T}_{\mathcal{R}}^{*} / F_{\text {cyc }}\right)$. Choose any $\xi \in \mathfrak{X}(\mathcal{R}) \backslash S_{J}$. It suffices to show the localization at $\mathcal{R} \backslash P_{\xi}, \mathcal{X}\left(\mathcal{T}_{\mathcal{R}} / F_{\text {cyc }}\right)_{\left(P_{\xi}\right)}$ $=0$. By (Tor) and Theorem 3.1, $\frac{\mathcal{X}\left(\mathcal{T}_{\mathcal{R}} / F_{\mathrm{cyc}}\right)}{P_{\xi} \mathcal{X}\left(\mathcal{T}_{\mathcal{R}} / F_{\mathrm{cyc}}\right)}$ is a torsion $\mathcal{R} / P_{\xi}$ module. From this, using localization argument and Nakayama's Lemma, we get $\mathcal{X}\left(\mathcal{T}_{\mathcal{R}} / F_{\text {cyc }}\right)_{\left(P_{\xi}\right)}=0$.

Proposition 3.6. Let $M$ and $N$ be two finitely generated torsion modules over the $r+1$ variable power series ring $R_{O}=O[[W, T]]$ where $W=\left(X_{1}, \cdots, X_{r}\right), O$ is the ring of integer of a finite extension of $\mathbb{Q}_{p}$. Let $\left\{l_{i}\right\}_{i \in \mathbb{N}}$ be an infinite set of co-height 1 prime ideals in $O[[W]]$ such that

(1) $O[[W]] / l_{i}$ is a finite extension of $O$, for any $i$.

(2) For each $l_{i}$, both $M / l_{i} M$ and $N / l_{i} N$ are torsion over $R_{O} / l_{i}$ and

(3) for every $i$, the image of $C h_{R_{O}}(M)$ (resp. $\left.C h_{R_{O}}(N)\right)$ in $R_{O} / l_{i}$ equals $C h_{R_{O} / l_{i}}\left(M / l_{i} M\right)$ (resp. $C h_{R_{O} / l_{i}}\left(N / l_{i} N\right)$ ), as ideals in $R_{O} / l_{i}$.

Then the equality of the ideals in $C h_{R_{O} / l_{i}}\left(M / l_{i} M\right)=C h_{R_{O} / l_{i}}\left(N / l_{i} N\right)$ in $R_{O} / l_{i}$ for every $l_{i}$ implies the equality of ideals $C h_{R_{O}}(M)=C h_{R_{O}}(N)$ in $R_{O}$.

Proof. For $r=1$, the result is essentially continued in [Oc3, §3]. Suppose $l_{i}=$ $\left(l_{i, 1}, l_{i, 2}, \cdots, l_{i, r}\right)$, denote by $l_{i}^{(j)}=\left(l_{i, 1}, l_{i, 2}, \cdots, l_{i, j}\right)$. Note that, $M \otimes R_{O} / l_{i}^{(j)}=(M \otimes$ $\left.R_{O} / l_{i}^{(j-1)}\right) \otimes R / l_{i, j}$. We claim that, if $C h_{R_{O} /\left(l_{i, 1}, l_{i, 2}, \cdots, l_{i, j}\right)} M \otimes R_{O} /\left(l_{i, 1}, l_{i, 2}, \cdots, l_{i, j}\right)=$ $C h_{R_{O} /\left(l_{i, 1}, l_{i, 2}, \cdots, l_{i, j}\right)} N \otimes R_{O} /\left(l_{i, 1}, l_{i, 2}, \cdots, l_{i, j}\right)$ is true for infinitely many $l_{i}^{(j)}$, for which first $(j-1)$ generators are same (that is $l_{i}^{(j-1)}$ 's are same for all $i$ ), then we have,

$$
C h_{R_{O} /\left(l_{i}^{(j-1)}\right)} M \otimes R_{O} /\left(l_{i}^{(j-1)}\right)=C h_{R_{O} /\left(l_{i}^{(j-1)}\right)} N \otimes R_{O} /\left(l_{i}^{(j-1)}\right) .
$$

Hence we prove the result by applying this to $j=r, r-1, \cdots, 1$.

We use multivariable notation $h(\mathbb{T})$ to denote polynomial $h\left(X_{1}, \cdots, X_{r}, T\right)$. As $M$ and $N$ are finitely generated torsion module over $(r+1)$ variable Iwasawa algebra, using the structure theorem of Iwasawa modules, we fix $R_{O}$ module pseudoisomorphisms $\phi$ and $\psi$ respectively,

$$
\begin{gathered}
M \stackrel{\phi}{\longrightarrow} \underset{i}{\oplus} R_{O} / \pi_{O}^{\mu_{i}} \underset{j}{\oplus} R_{O} / h_{j}(\mathbb{T})^{\lambda_{j}} \text { and } \\
N \stackrel{\psi}{\longrightarrow} \underset{i^{\prime}}{\oplus} R_{O} / \pi_{O}^{\mu_{i^{\prime}}^{\prime}} \underset{j^{\prime}}{\oplus} R_{O} / g_{j^{\prime}}(\mathbb{T})^{\lambda_{j^{\prime}}^{\prime}} .
\end{gathered}
$$

Here $\pi_{O}$ is a uniformizing parameter for $O$. Set $h(\mathbb{T})=\prod h_{j}(\mathbb{T})^{\lambda_{j}}, g(\mathbb{T})=\prod g_{j^{\prime}}(\mathbb{T})^{\lambda_{j^{\prime}}}$ and $\mu=\sum \mu_{i}, \mu^{\prime}=\sum \mu_{i^{\prime}}$. 
We will show that $C h_{R_{O} / l_{i}^{(j-1)}}\left(N \otimes R_{O} / l_{i}^{(j-1)}\right) \subset C h_{R_{O} / l_{i}^{(j-1)}}\left(M \otimes R_{O} / l_{i}^{(j-1)}\right)$. Interchanging $M$ and $N$, we will get the equality. Clearly, it suffices to show that the image of $\pi_{O}^{\mu^{\prime}}$ is zero in $\left(R_{O} / l_{i}^{(j-1)}\right) / \pi_{O}^{\mu}$ and the image of $g(\mathbb{T})$ is zero in $\left(R_{O} / l_{i}^{(j-1)}\right) / h(\mathbb{T})$.

If $h(\mathbb{T})$ is a unit in $R_{O} / l_{i}^{(j-1)}$ then obviously the image of $g(\mathbb{T})$ in $\left(R_{O} / l_{i}^{(j-1)}\right) / h(\mathbb{T})$ is zero. So we assume that $h(\mathbb{T})$ is not a unit in $R_{O} / l_{i}^{(j-1)}$. Then by [Oc3, Lemma 3.8] there is a finite extension $O^{\prime \prime}$ of $O$ such that by a change of coordinate by a linear transform, we may assume that $h(\mathbb{T})=u(\mathbb{T}) f(T)$ where $u(\mathbb{T})$ is a unit in $R_{O^{\prime \prime}}$ and $f \in O^{\prime \prime}[[W]][T]$. Now, if necessary, we move to an extension of $O^{\prime \prime}$ containing both $O^{\prime}$ and $O^{\prime \prime}$ and denote again it by $O^{\prime}$ (abusing the notation, just to ease the burden of notation) such that $R_{O^{\prime}} / l_{i} \cong O^{\prime}[[T]]$. Then, the image of $g(\mathbb{T})$ vanishes in $R_{O^{\prime}} /\left(h(\mathbb{T}), l_{i}^{(j)}\right)$ for every $i$.

For every $k \geq 1$, we have an injection

$$
\left(R_{O^{\prime}} / l_{i}^{(j-1)}\right) /\left(l_{1, j} l_{2, j} \cdots l_{k, j}\right) \hookrightarrow \prod_{1 \leq i \leq k}\left(R_{O^{\prime}} / l_{i}^{(j-1)}\right) /\left(l_{i, j}\right) \cong \prod_{1 \leq i \leq k} R_{O^{\prime}} / l_{i}^{(j)}
$$

Since $R_{O^{\prime}} / h(\mathbb{T})$ is finite flat over $O^{\prime}[[W]]$, we get for each $k \geq 1$ an injection,

$$
\left(R_{O^{\prime}} / l_{i}^{(j-1)}\right) /\left(h(\mathbb{T}), l_{1, j} l_{2, j} \cdots l_{k, j}\right) \hookrightarrow \prod_{1 \leq i \leq k} R_{O^{\prime}} /\left(h(\mathbb{T}), l_{i}^{(j)}\right) .
$$

We observe that image of $g(\mathbb{T})$ vanishes in $\left(R_{O^{\prime}} / l_{i}^{(j-1)}\right) /\left(h(\mathbb{T}), l_{1, j} l_{2, j} \cdots l_{k, j}\right)$. Thus the image of $g(\mathbb{T})$ is zero in $R_{O^{\prime}} /\left(h(\mathbb{T}), l_{i}^{(j-1)}\right)$, since

$$
\lim _{k}\left(R_{O^{\prime}} / l_{i}^{(j-1)}\right) /\left(h(\mathbb{T}), l_{1, j} l_{2, j} \cdots l_{k, j}\right) \cong R_{O^{\prime}} /\left(h(\mathbb{T}), l_{i}^{(j-1)}\right) .
$$

For the $\mu$ invariants, for any $l_{i}, \pi_{O^{\prime}}^{\mu}$ (resp. $\pi_{O^{\prime}}^{\mu^{\prime}}$ ) is equal to the highest power of $\pi_{O}$ dividing the characteristic power series of $M_{O^{\prime}} / l_{i} M_{O^{\prime}}$ (resp. $N_{O^{\prime}} / l_{i} N_{O^{\prime}}$ ), where $M_{O^{\prime}}:=M \otimes_{O} O^{\prime}$ is the extension of scalers from $O$ to $O^{\prime}$. Hence $\pi_{O^{\prime}}^{\mu}=\pi_{O^{\prime}}^{\mu^{\prime}}$. Thus it follows that $\pi_{O}^{\mu^{\prime}}$ is zero in $\left(R_{O^{\prime}} / l_{i}^{(j-1)}\right) / \pi_{O}^{\mu}$.

Proposition 3.7. For any finitely generated $\mathcal{R}[[\Gamma]]$ module $U$, let $U^{0}$ denotes the maximal pseudonull $\mathcal{R}[[\Gamma]]$ submodule of $U$. Assume the hypothesis (Tor). Then for every $\xi \in \mathfrak{X}(\mathcal{R}), \frac{\mathcal{X}\left(\mathcal{T}_{\mathcal{R}} / F_{\mathrm{cyc}}\right)^{0}}{P_{\xi} \mathcal{X}\left(\mathcal{T}_{\mathcal{R}} / F_{\mathrm{cyc}}\right)^{0}}$ (resp. $\left.\frac{\mathcal{X}\left(\mathcal{T}_{\mathcal{R}}^{*} / F_{\mathrm{cyc}}\right)^{0}}{P_{\xi} \mathcal{X}\left(\mathcal{T}_{\mathcal{R}}^{*} / F_{\mathrm{cyc}}\right)^{0}}\right)$ are pseudonull $O_{f_{\xi}}[[\Gamma]]$ modules.

Proof. We broadly follow the same strategy as in [Oc1, Lemma 7.2] but the arguments are different. We prove the result only for $\mathcal{X}\left(\mathcal{T}_{\mathcal{R}} / F_{\text {cyc }}\right)$ as an entirely similar argument holds for $\mathcal{X}\left(\mathcal{T}_{\mathcal{R}}^{*} / F_{\text {cyc }}\right)$. Recall, for $C=\mathcal{A}$ or $C=A_{f_{\xi}}$, with notation as before, the Selmer group $S\left(C / F_{\text {cyc }}\right)$ fits into the exact sequence

$$
0 \rightarrow S\left(C / F_{\mathrm{cyc}}\right) \longrightarrow H^{1}\left(F_{S} / F_{\mathrm{cyc}}, C\right) \longrightarrow \underset{v_{c} \mid S, v_{c} \nmid p}{\oplus} H^{1}\left(I_{v_{c}}, C\right)^{G_{v_{c}}} \underset{v_{c}|\mathfrak{p}| p}{\oplus} H^{1}\left(I_{v_{c}}, C_{\mathfrak{p}}^{-}\right)^{G_{v_{c}}}
$$

Note, for $v_{c} \nmid p$,

$$
0 \rightarrow H^{1}\left(G_{v_{c}} / I_{v_{c}}, C^{I_{v_{c}}}\right) \longrightarrow H^{1}\left(G_{v_{c}}, C\right) \underset{22}{\longrightarrow} H^{1}\left(I_{v_{c}}, C\right)^{G_{v_{c}}} \longrightarrow H^{2}\left(G_{v_{c}} / I_{v_{c}}, C^{I_{v_{c}}}\right)
$$


with $G_{v_{c}} / I_{v_{c}} \cong \underset{l \neq p}{\oplus} \mathbb{Z}_{l}$ and $C$ is a $p$-torsion group. Thus $H^{i}\left(G_{v_{c}} / I_{v_{c}}, C^{I_{v_{c}}}\right)=0$ for $i=1,2$. Thus we see that, $H^{1}\left(G_{v_{c}}, C\right) \simeq H^{1}\left(I_{v_{c}}, C\right)^{G_{v_{c}}}$.

Combining these, we can have the following alternative definition of $S\left(C / F_{\text {cyc }}\right)$.

$$
0 \rightarrow S\left(C / F_{\mathrm{cyc}}\right) \longrightarrow H^{1}\left(F_{S} / F_{\mathrm{cyc}}, C\right) \longrightarrow \underset{v_{c} \mid S, v_{c} \nmid p}{\oplus} H^{1}\left(G_{v_{c}}, C\right) \underset{v_{c}|\mathfrak{p}| p}{\oplus} H^{1}\left(I_{v_{c}}, C_{\mathfrak{p}}^{-}\right)^{G_{v_{c}}}
$$

Similarly, for strict Selmer group we obtain that,

$$
0 \rightarrow S^{s t r}\left(C / F_{\mathrm{cyc}}\right) \longrightarrow H^{1}\left(F_{S} / F_{\mathrm{cyc}}, C\right) \longrightarrow \underset{v_{c} \mid S, v_{c} \nmid p}{\oplus} H^{1}\left(G_{v_{c}}, C\right) \underset{v_{c}|\mathfrak{p}| p}{\oplus} H^{1}\left(G_{v_{c}}, C_{\mathfrak{p}}^{-}\right)
$$

We have from $[\mathrm{F}-\mathrm{O}, \S 2.2 .2]$ an exact sequence,

$$
0 \rightarrow S^{s t r}\left(C / F_{\text {cyc }}\right) \rightarrow S\left(C / F_{\text {cyc }}\right) \rightarrow \underset{\mathfrak{p} \mid p}{\oplus} H^{1}\left(G_{F_{\mathfrak{p}}} / I_{\mathfrak{p}},\left(C_{\mathfrak{p}}^{-}\right)^{I_{\mathfrak{p}}}\right)
$$

Moreover from the proof of [F-O, Corollary 3.4], when $C=\mathcal{A}$, we see that $S^{s t r}\left(\mathcal{A} / F_{\text {cyc }}\right)=S\left(\mathcal{A} / F_{\text {cyc }}\right)$.

Under the assumption (Tor), we have that $X\left(T_{f_{\xi}} / F_{\text {cyc }}\right)$ is torsion for any $\xi \in$ $\mathfrak{X}(\mathcal{R})$. Also by corollary $3.5, \mathcal{X}\left(\mathcal{T}_{\mathcal{R}} / F_{\text {cyc }}\right)$ is torsion over $\mathcal{R}[[\Gamma]]$. It follows that the maps defining $S\left(C / F_{\text {cyc }}\right)$ in (45) is surjective for $C=\mathcal{A}$ or $C=A_{f_{\xi}}$ with $\xi \in \mathfrak{X}(\mathcal{R})([\mathrm{Oc} 1$, Corollary 4.12]). Now let us consider the commutative diagram

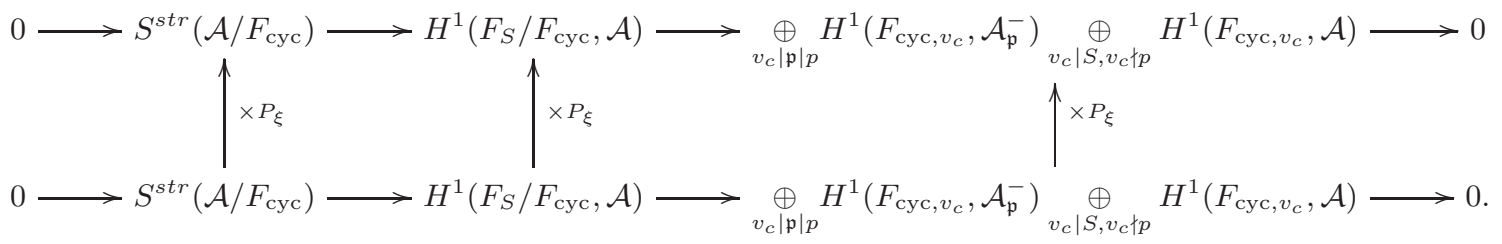

Recall $A_{f_{\xi}} \cong \mathcal{A}\left[P_{\xi}\right]$ and $\left(A_{f_{\xi}}\right)_{\mathfrak{p}}^{-} \cong \mathcal{A}_{\mathfrak{p}}^{-}\left[P_{\xi}\right]$ as Galois modules. For any $\xi \in \mathfrak{X}(\mathcal{R})$, as $X\left(T_{f_{\xi}^{*}} / F_{\text {cyc }}\right)$ is torsion by (Tor), we get that $H^{2}\left(F_{S} / F_{\text {cyc }}, A_{f_{\xi}}\right)=0$ (see [H-M, Proposition 2.3]). Then the cokernel of the middle vertical map in (46), being is a subgroup of $H^{2}\left(F_{S} / F_{\text {cyc }}, A_{f_{\xi}}\right)$, vanishes. Thus by applying a Snake lemma to the diagram (46), we get that

$$
\frac{S^{s t r}\left(\mathcal{A} / F_{\mathrm{cyc}}\right)}{P_{\xi} S^{s t r}\left(\mathcal{A} / F_{\mathrm{cyc}}\right)} \cong \operatorname{coker}\left(H^{1}\left(F_{S} / F_{\mathrm{cyc}}, \mathcal{A}\right)\left[P_{\xi}\right] \stackrel{l_{\xi}}{\longrightarrow} W\left[P_{\xi}\right]\right)
$$

where

$$
W:=\underset{v_{c}|\mathfrak{p}| p}{\bigoplus} H^{1}\left(F_{\mathrm{cyc}, v_{c}}, \mathcal{A}_{\mathfrak{p}}^{-}\right) \underset{v_{c} \mid S, v_{c} \nmid p}{\bigoplus} H^{1}\left(F_{\mathrm{cyc}, v_{c}}, \mathcal{A}\right) .
$$

Similarly define

$$
W_{\xi}:=\underset{v_{c}|\mathfrak{p}| p}{\bigoplus} H^{1}\left(F_{\mathrm{cyc}, v_{c}},\left(A_{f_{\xi}}\right)_{\mathfrak{p}}^{-}\right) \underset{v_{c} \mid S, v_{c} \nmid p}{\bigoplus} H^{1}\left(F_{\mathrm{cyc}, v_{c}}, A_{f_{\xi}}\right) .
$$

Then we have the commutative diagram

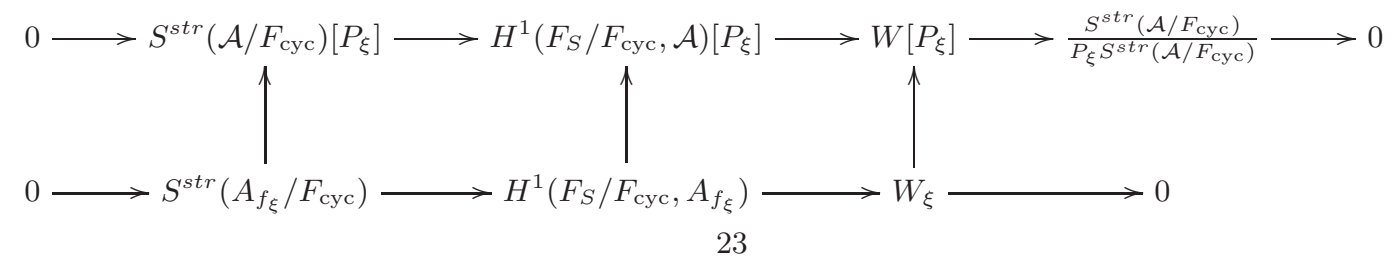


We see that the natural map $W_{\xi} \longrightarrow W\left[P_{\xi}\right]$ above is surjective. From the diagram (47), we see that the natural map $\left.H^{1}\left(F_{S} / F_{\text {cyc }}, \mathcal{A}\right)\left[P_{\xi}\right] \stackrel{l_{\xi}}{\longrightarrow} W\left[P_{\xi}\right]\right)$ is surjective. Thus we obtain $S^{s t r}\left(\mathcal{A} / F_{\text {cyc }}\right) / P_{\xi} S^{s t r}\left(\mathcal{A} / F_{\text {cyc }}\right)=0$. Since $S^{s t r}\left(\mathcal{A} / F_{\text {cyc }}\right)=S\left(\mathcal{A} / F_{\text {cyc }}\right)$, we see that, $S\left(\mathcal{A} / F_{\text {cyc }}\right) / P_{\xi} S\left(\mathcal{A} / F_{\text {cyc }}\right)=0$. In other words, $\mathcal{X}\left(\mathcal{T}_{\mathcal{R}} / F_{\text {cyc }}\right)\left[P_{\xi}\right]=0$. Thus $\mathcal{X}\left(\mathcal{T}_{\mathcal{R}} / F_{\text {cyc }}\right)^{0}\left[P_{\xi}\right]=0$. In particular, $\mathcal{X}\left(\mathcal{T}_{\mathcal{R}} / F_{\text {cyc }}\right)^{0}\left[P_{\xi}\right]$ is a pseudonull $O_{f_{\xi}}[[\Gamma]]$ module. But for any finitely generated pseudonull $\mathcal{R}[[\Gamma]]$ module $M, M / P_{\xi}$ is a pseudonull $O_{f_{\xi}}[[\Gamma]]$ module if and only if $M\left[P_{\xi}\right]$ is so ([Oc3, Lemma 3.1]). Thus, the last fact in turn implies that $\frac{\mathcal{X}\left(\mathcal{T}_{\mathcal{R}} / F_{\text {cyc }}\right)^{0}}{P_{\xi} \mathcal{X}\left(\mathcal{T}_{\mathcal{R}} / F_{\text {cyc }}\right)^{0}}$ is also a pseudonull $O_{f_{\xi}}[[\Gamma]]$ module.

Lemma 3.8. Let $J$ be any non-zero ideal in $\mathcal{R}$. Let $I \in \operatorname{Spec}(\mathcal{R}) \backslash \operatorname{Spec}(\mathcal{R} / J)$ (that is $I$ is a prime ideal in $\mathcal{R}$, which does not contain $J$ ). There exists at most finitely many $z_{1}, \cdots, z_{k}$ in $\mathcal{R}$ with the following properties:

(1) $\left(I, z_{i}\right)$ are distinct prime ideals in $\mathcal{R}$ for all $i=1, \cdots, k$.

(2) For $i \neq j, \bar{z}_{i} \nmid \bar{z}_{j}$ in $\mathcal{R} / I$.

(3) $\left(I, z_{i}\right) \supset J$ for all $i=1, \cdots, k$.

Proof. First we claim that,

$$
\cap_{i=1}^{r}\left(I, z_{i}\right)=\left(I, z_{1} z_{2} \cdots z_{r}\right) .
$$

Obviously, $\left(I, z_{1} z_{2} \cdots z_{r}\right) \subseteq \cap_{i=1}^{r}\left(I, z_{i}\right)$. Now, let $x \in \cap_{i=1}^{r}\left(I, z_{i}\right)$. Then,

$$
x=i_{1}+a_{1} z_{1}=\cdots=i_{r}+a_{r} z_{r},
$$

with $i_{j} \in I$ and $a_{j} \in \mathcal{R}$. Then we see that,

$$
\bar{x}=\overline{a_{1}} \overline{z_{1}}=\cdots=\overline{a_{r}} \overline{z_{r}} \in \mathcal{R} / I \text {. }
$$

Since $\bar{z}_{i} \nmid \bar{z}_{j}$, we see that $\bar{x}=\bar{\alpha} \bar{z}_{1} \cdots \bar{z}_{r} \in \mathcal{R} / I$. Thus, $x=i+\alpha z_{1} \cdots z_{r}$, where $i \in I$ and $\alpha$ is some lift of $\bar{\alpha}$ in $\mathcal{R}$, which completes the proof of the claim.

Suppose that there are infinitely many $z_{i}$ 's in $\mathcal{R}$ which satisfies all three properties. Then we have a decreasing chain of ideals in $\mathcal{R}$,

$$
\left(I, z_{1}\right) \supset\left(I, z_{1} z_{2}\right)=\cap_{i=1}^{2}\left(I, z_{i}\right) \supset \cdots \supset\left(I, z_{1} \cdots z_{r}\right)=\cap_{i=1}^{r}\left(I, z_{i}\right) \supset \cdots .
$$

By assumption, $\left(I, z_{i}\right)$ contains the ideal $J$ for all $i$. Thus we obtain,

$$
J \subseteq \cap_{i=1}^{\infty}\left(I, z_{i}\right)=\lim _{r}\left(I, z_{1} \cdots z_{r}\right) \cong I,
$$

which is a contradiction to our assumption $I \in \operatorname{Spec}(\mathcal{R}) \backslash \operatorname{Spec}(\mathcal{R} / J)$.

Remark 3.9. Let $M=\mathcal{X}\left(\mathcal{T}_{\mathcal{R}} / F_{\text {cyc }}\right)$ and $N=\mathcal{X}\left(\mathcal{T}_{\mathcal{R}}^{*} / F_{\text {cyc }}\right)^{\iota}$. Assume (Irr), (Dist), (Tor) and $\mathcal{R} \cong O[[W]]$. Let $S_{1}$ be the subset of arithmetic points for which $f_{\xi}$ 's are exceptional (as defined in Theorem 2.3). Also let $S_{2}$ be the subset of $\mathfrak{X}(\mathcal{R})$ for which at least one among $\operatorname{ker}\left(s_{\xi}\right), \operatorname{coker}\left(s_{\xi}\right), \operatorname{ker}\left(s_{\xi}^{*}\right)$ and coker $\left(s_{\xi}^{*}\right)$ associated to the the natural specialization map $s_{\xi}$ in Theorem 3.1 and $s_{\xi}^{*}$ in remark 3.2 is infinite. Define $S=S_{1} \cup S_{2}$. Put $\mathfrak{X}(\mathcal{R})^{\prime}:=\mathfrak{X}(\mathcal{R}) \backslash S$, then $\mathfrak{X}(\mathcal{R})^{\prime}$ is infinite.

Take the set $\left\{l_{i}\right\}_{i \in \mathbb{N}}=\mathfrak{X}(\mathcal{R})^{\prime}$. Then from Theorem 3.1, corollary 3.5, Proposition 3.7 and Lemma 3.8, we deduce that for these choices of $M, N$ and $l_{i}$ 's, all the condition of Proposition 3.6 are satisfied (Lemma 3.8 ensures us that for each $j$ in 
the proof of Proposition 3.6, we have infinitely many ideals $l_{i}^{(j)}$ for which first $(j-1)$ generators are the same).

Theorem 3.10. Let the notation be as before. Let $F$ be a totally real number field, with $\Gamma=\operatorname{Gal}\left(F_{\text {cyc }} / F\right) \cong \mathbb{Z}_{p}$. Assume

(1) (Irr): The residual representation $\bar{\rho}_{\mathcal{R}}$ of $G_{F}$ is absolutely irreducible.

(2) (Dist): The restriction of the residual representation at the decomposition subgroup i.e. $\left.\bar{\rho}_{\mathcal{R}}\right|_{G_{\mathfrak{p}}}$ is an extension of two distinct characters of $G_{\mathfrak{p}}$ with values in $\mathbb{F}_{\mathcal{R}}^{*}$ for each $\mathfrak{p} \mid p$.

(3) (Tor): For any normalized cuspidal Hilbert eigenform $f, X\left(T_{f} / F_{\text {cyc }}\right)$ is a finitely generated torsion $O_{f}[[\Gamma]]$ module.

(4) $\mathcal{R}$ is a power series ring.

Then the functional equation holds for $\mathcal{X}\left(\mathcal{T}_{\mathcal{R}} / F_{\text {cyc }}\right)$ i.e. as an ideal in $\mathcal{R}[[\Gamma]]$,

$$
C h_{\mathcal{R}[[\Gamma]]}\left(\mathcal{X}\left(\mathcal{T}_{\mathcal{R}} / F_{\text {cyc }}\right)\right)=C h_{\mathcal{R}[[\Gamma]]}\left(\mathcal{X}\left(\mathcal{T}_{\mathcal{R}}^{*} / F_{\text {cyc }}\right)^{\iota}\right)
$$

Proof. By corollary $3.5, \mathcal{X}\left(\mathcal{T}_{\mathcal{R}} / F_{\text {cyc }}\right)$ and $\mathcal{X}\left(\mathcal{T}_{\mathcal{R}}^{*} / F_{\text {cyc }}\right)$ are torsion $\mathcal{R}[[\Gamma]]$ modules. Using remark 3.9 , choose the infinite subset $\mathfrak{X}(\mathcal{R})^{\prime}$ of arithmetic points. By corollary 3.5 , for every $\xi \in \mathfrak{X}(\mathcal{R})^{\prime}, X\left(T_{f_{\xi}} / F_{\text {cyc }}\right)$ and $X\left(T_{f_{\xi}}^{*} / F_{\text {cyc }}\right)^{\iota}$ are torsion over $O_{f_{\xi}}[[\Gamma]]$.

Then applying Proposition 3.6 for $M=\mathcal{X}\left(\mathcal{T}_{\mathcal{R}} / F_{\text {cyc }}\right), N=\mathcal{X}\left(\mathcal{T}_{\mathcal{R}}^{*} / F_{\text {cyc }}\right)^{\iota}$ and $\left\{l_{i}\right\}_{i \in \mathbb{N}}=$ $\mathfrak{X}(\mathcal{R})^{\prime}$, to prove the theorem it suffices to show that for every $\xi \in \mathfrak{X}(\mathcal{R})^{\prime}$,

$$
C h_{O_{f_{\xi}}[[\Gamma]]}\left(\frac{\mathcal{X}\left(\mathcal{T}_{\mathcal{R}} / F_{\text {cyc }}\right)}{P_{\xi} \mathcal{X}\left(\mathcal{T}_{\mathcal{R}} / F_{\text {cyc }}\right)}\right)=C h_{O_{f_{\xi}}[[\Gamma]]}\left(\frac{\mathcal{X}\left(\mathcal{T}_{\mathcal{R}}^{*} / F_{\text {cyc }}\right)^{\iota}}{P_{\xi} \mathcal{X}\left(\mathcal{T}_{\mathcal{R}}^{*} / F_{\text {cyc }}\right)^{\iota}}\right)
$$

considered as ideals in $O_{f_{\xi}}[[\Gamma]]$. By Theorem 3.1, remark 3.2 and remark 3.3 this in turn equivalent to showing

$$
C h_{\left.O_{f_{\xi}}[\Gamma]\right]}\left(X\left(T_{f_{\xi}} / F_{\text {cyc }}\right)\right)=C h_{O_{f_{\xi}}[[\Gamma]]}\left(X\left(T_{f_{\xi}}^{*} / F_{\text {cyc }}\right)^{\iota}\right)
$$

for each $\xi \in \mathfrak{X}(\mathcal{R})^{\prime}$. Hence we are done by Theorem 2.10.

\section{ReSUlts OVER $\mathbb{Z}_{p}^{2}$ EXTENSION}

Let $K_{\infty} / K$ be the unique $\mathbb{Z}_{p}^{\oplus 2}$ extension of an imaginary quadratic field $K$. In this section, we will assume throughout that $p$ splits in $K$ and $D_{K}$ the discriminate of the imaginary quadratic field $K$, is coprime to tame conductor $N_{\mathcal{R}}$ of the branch $\mathcal{R}$ of the Hida family i.e. $\left(p, D_{K}\right)=\left(D_{K}, N_{\mathcal{R}}\right)=\left(N_{\mathcal{R}}, p\right)=1$. Recall the notation, $\Gamma_{K}=\operatorname{Gal}\left(K_{\infty} / K\right) \cong \mathbb{Z}_{p}^{2}, \Gamma=G\left(K_{\text {cyc }} / K\right) \cong \mathbb{Z}_{p}$ and $H=\operatorname{Gal}\left(K_{\infty} / K_{\text {cyc }}\right) \cong \mathbb{Z}_{p}$ so that $G / H \cong \Gamma$.

Recall from remark 1.21, $S=S_{K}$ is a finite set of primes of $K$ dividing $N p$. Let

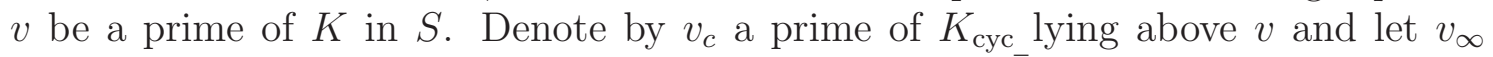
be a prime of $K_{\infty}$ lying above $v_{c}$. Let $\bar{v}$ be a prime of $\overline{\mathbb{Q}}$ lying above a prime $v_{\infty}$. Let $G_{v_{c}}$ and $G_{v_{\infty}}$ denotes the decomposition subgroup of $\overline{\mathbb{Q}} / K_{\text {cyc }}$ and $\overline{\mathbb{Q}} / K_{\infty}$ for the prime $\bar{v} / v_{c}$ and $\bar{v} / v_{\infty}$ respectively. Let $I_{v_{c}}, I_{v_{\infty}}$ denote the inertia group of $\overline{\mathbb{Q}} / K_{\text {cyc }}$ and $\overline{\mathbb{Q}} / K_{\infty}$ for the prime $\bar{v} / v_{c}$ and $\bar{v} / v_{\infty}$ respectively. Let $\Gamma_{K_{v}}$ (resp. $\left.H_{v_{c}}\right)$ denote the decomposition subgroup of $\Gamma_{K}$ (resp. $H$ ) with respect to primes $v_{\infty} / v$ (resp. $\left.v_{\infty} / v_{c}\right)$. We write $I_{v_{\infty} / v_{c}}$ for the inertia subgroup of $K_{\infty} / K_{\text {cyc }}$ with respect to prime $v_{\infty} / v_{c}$ 
Proposition 4.1. The kernel of the map

$$
X\left(T_{f} / K_{\infty}\right)_{H} \stackrel{\alpha_{H}^{\vee}}{\longrightarrow} X\left(T_{f} / K_{\text {cyc }}\right)
$$

is a finitely generated $\mathbb{Z}_{p}$ module and the cokernel of $\alpha_{H}^{\vee}$ is finite.

Proof. Set

$$
\begin{gathered}
J_{v_{c}}:= \begin{cases}H^{1}\left(I_{v_{c}}, A_{f}\right)^{G_{v_{c}}} & \text { if } v_{c} \mid S, v_{c} \nmid p \\
H^{1}\left(I_{v_{c}}, A_{f}^{-}\right)^{G_{v_{c}}} & \text { if } v_{c} \mid p,\end{cases} \\
J_{v_{c}}^{\infty}:= \begin{cases}\prod_{v_{\infty} \mid v_{c}} H^{1}\left(I_{v_{\infty}}, A_{f}\right)^{G_{v_{\infty}}} & \text { if } v_{c} \mid S, v_{c} \nmid p \\
\prod_{v_{\infty} \mid v_{c}} H^{1}\left(I_{v_{\infty}}, A_{f}^{-}\right)^{G_{v_{\infty}}} & \text { if } v_{c} \mid p,\end{cases}
\end{gathered}
$$

Using similar argument as in (43), we get that

$$
J_{v_{c}}:= \begin{cases}H^{1}\left(K_{\mathrm{cyc}, v_{c}}, A_{f}\right) & \text { if } v_{c} \mid S, v_{c} \nmid p \\ H^{1}\left(I_{v_{c}}, A_{f}^{-}\right)^{G_{v_{c}}} & \text { if } v_{c} \mid p\end{cases}
$$

Also,

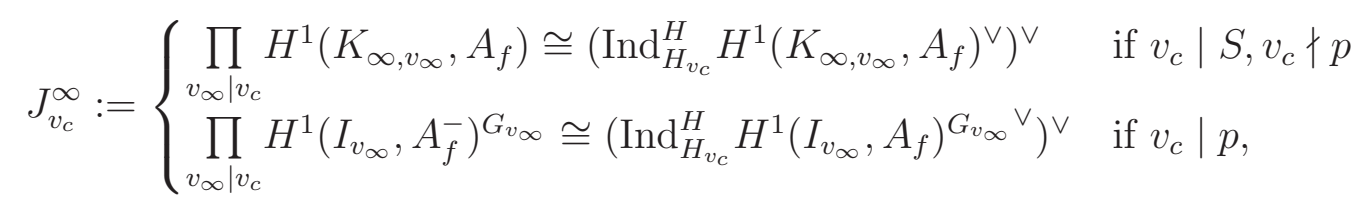

Then we have the commutative diagram

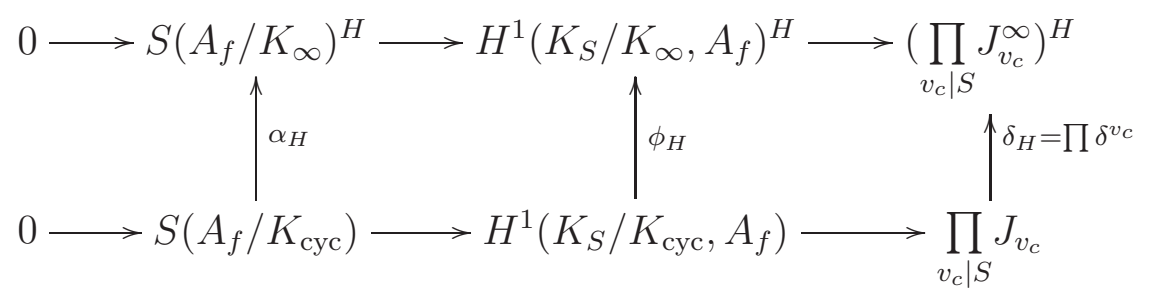

By inflation-restriction sequence of Galois cohomology, the kernel of $\phi_{H}$ is isomorphic to $H^{1}\left(H, A_{f}^{G_{K \infty}}\right)$. Clearly $U_{\infty}:=A_{f}^{G_{K_{\infty}} \vee}$ is a finitely generated $\mathbb{Z}_{p}$ module. Thus we deduce that $\operatorname{ker}\left(\phi_{H}\right)^{\vee}$ is a finitely generated $\mathbb{Z}_{p}$ module. Moreover, as $H \cong \mathbb{Z}_{p}$, we see that $H^{1}\left(H, A_{f}^{G_{K_{\infty}}}\right)$ is finite if and only if $H^{0}\left(H, A_{f}^{G_{K_{\infty}}}\right) \cong A_{f}^{G_{K_{c y c}}}$ is finite. The last fact follows from (cf. [C-S, Theorem A 2.8], [Su, Lemma 2.1]). Hence, by Snake lemma on diagram (48), we deduce that $\operatorname{cocker}\left(\alpha_{H}^{\vee}\right)$ is finite.

Also as $H$ has $p$-cohomological dimension $=1, \phi_{H}$ is surjective. Thus $\operatorname{ker}\left(\alpha_{H}\right)^{\vee}$ is a subquotient of the Pontryagin dual of kernel of $\delta_{H}$. Given a prime $v_{c}$ we pick any one prime $v_{\infty}$ in $K_{\infty}$. Then by Shapiro's Lemma for each $v_{c}$,

$$
H^{1}\left(H, J_{v_{c}}^{\infty}\right) \cong \begin{cases}H^{1}\left(K_{\infty, v_{\infty}}, A_{f}\right) & \text { if } v_{c} \mid S, v_{c} \nmid p \\ H^{1}\left(I_{v_{\infty}}, A_{f}^{-}\right)^{G_{v_{\infty}}} & \text { if } v_{c} \mid p\end{cases}
$$

Then 


$$
\operatorname{ker}\left(\delta^{v_{c}}\right) \cong \begin{cases}H^{1}\left(H_{v_{c}}, A_{f}^{G_{v_{\infty}}}\right) & \text { if } v_{c} \mid S, v_{c} \nmid p \\ \text { a subgroup of } H^{1}\left(I_{v_{\infty} / v_{c}}, A_{f}^{-I_{v_{\infty}}}\right) & \text { if } v_{c} \mid p\end{cases}
$$

where the inertia subgroup $I_{v_{\infty} / v_{c}} \cong \mathbb{Z}_{p}$. As before, we conclude that the Pontryagin dual of $\operatorname{ker}\left(\delta^{v_{c}}\right)$ is a finitely generated $\mathbb{Z}_{p}$ module. From these, summing over finitely many primes and using a Snake lemma on diagram (48), we deduce that $\operatorname{ker}\left(\alpha_{H}^{\vee}\right)$ is finitely generated over $\mathbb{Z}_{p}$.

Remark 4.2. By Kato's result (see [Ka]), we know that for any p-stabilized newform $f, X\left(T_{f} / K_{\mathrm{cyc}}\right)$ (and $X\left(T_{f}^{*} / K_{\mathrm{cyc}}\right)$ ) are finitely generated torsion $\mathbb{Z}_{p}[[\Gamma]]$ modules.

Corollary 4.3. For any $p$-stabilized newform $f, X\left(T_{f} / K_{\infty}\right)$ and $X\left(T_{f}^{*} / K_{\infty}\right)$ are finitely generated torsion $O_{f}\left[\left[\Gamma_{K}\right]\right]$ modules.

Proof. By remark 4.2 , we have $X\left(T_{f} / K_{\text {cyc }}\right)$ is a finitely generated torsion $O_{f}[[\Gamma]]$ module. By Proposition 4.1, we also have an exact sequence

$$
0 \longrightarrow F_{1} \longrightarrow X\left(T_{f} / K_{\infty}\right)_{H} \longrightarrow X\left(T_{f} / K_{\mathrm{cyc}}\right) \longrightarrow F_{2} \longrightarrow 0
$$

with $F_{1}$ is a finitely generated $\mathbb{Z}_{p}$ modules and $F_{2}$ is finite. Thus we get that $X\left(T_{f} / K_{\infty}\right)_{H}$ is a finite generated torsion $O_{f}[[\Gamma]]$ module. But for an ideal $I \neq$ $O_{f}\left[\left[\Gamma_{K}\right]\right]$ in $O_{f}\left[\left[\Gamma_{K}\right]\right]$

$$
\operatorname{rank}_{\frac{O_{f}\left[\left[\Gamma_{K}\right]\right]}{I}} \frac{X\left(T_{f} / K_{\infty}\right)}{I X\left(T_{f} / K_{\infty}\right)} \geq \operatorname{rank}_{O_{f}\left[\left[\Gamma_{K}\right]\right]} X\left(T_{f} / K_{\infty}\right) .
$$

Identifying $O_{f}\left[\left[\Gamma_{K}\right]\right]$ with $O_{f}\left[\left[T_{1}, T_{2}\right]\right]$ and $X\left(T_{f} / K_{\infty}\right)_{H}$ with $\frac{X\left(T_{f} / K_{\infty}\right)}{T_{2} X\left(T_{f} / F_{\infty}\right)}$, we deduce that $\operatorname{rank}_{O_{f}\left[\left[\Gamma_{K}\right]\right]} X\left(T_{f} / K_{\infty}\right)=0$. The argument for $X\left(T_{f}^{*} / F_{\infty}\right)$ is similar.

Definition 4.4. We call $f \in S_{2}\left(\Gamma_{0}(N p), \psi\right)$ exceptional if $f$ is a newform of conductor $N p$ with $(N, p)=($ conductor of $\psi, p)=1$.

Theorem 4.5. Let the notation be as before. Let $f \in S_{k}\left(\Gamma_{0}\left(N p^{r}\right), \psi\right)$ be a pstabilized newform which is not exceptional. Also assume that $\left(N, D_{K}\right)=\left(p, D_{K}\right)=$ 1. Then the functional equation holds for $X\left(T_{f} / K_{\infty}\right)$ i.e. we have a equality of ideals in $O_{f}\left[\left[\Gamma_{K}\right]\right]$,

$$
C h_{O_{f}\left[\left[\Gamma_{K}\right]\right]}\left(X\left(T_{f} / K_{\infty}\right)\right)=C h_{O_{f}\left[\left[\Gamma_{K}\right]\right]}\left(X\left(T_{f}^{*} / K_{\infty}\right)^{\iota}\right) .
$$

Proof. Note as the $p$-ordinary, $p$-stabilized newform $f$ is not exceptional and we have, $\left(N, D_{K}\right)=\left(p, D_{K}\right)=1$; the Galois representation $\left(\rho_{f}, V_{f}\right)$ satisfies both $\left(\operatorname{Hyp}\left(K_{\infty}, V_{f}\right)\right)$ and $\left(\operatorname{Hyp}\left(K_{\infty}, V_{f}^{\vee}\right)\right)$ assumptions of [Pe, Theorem 4.2.1]. Also, by corollary 4.3 , we get that both $X\left(T_{f} / K_{\infty}\right)$ and $X\left(T_{f}^{*} / K_{\infty}\right)^{\iota}$ are torsion over $O_{f}\left[\left[\Gamma_{K}\right]\right]$. Thus the condition $\left(\operatorname{Tors}\left(K_{\infty}, V_{f}\right)\right)$ in [Pe, Theorem 4.2.1] is also satisfied. Hence the theorem follows from [Pe, Theorem 4.2.1].

Let us recall from remark 1.12, $\mathcal{T}_{\mathcal{R}}$ is a lattice associated to a fixed branch $\mathcal{R}$ of the ordinary Hida family of elliptic modular form of tame level $N=N_{\mathcal{R}}$. 
Proposition 4.6. Assume (Irr), (Dist) and $\mathcal{R}$ is a power series ring. Then the kernel and the cokernel of the natural specialization map

$$
\mathcal{X}\left(\mathcal{T}_{\mathcal{R}} / K_{\infty}\right) / P_{\xi} \mathcal{X}\left(\mathcal{T}_{\mathcal{R}} / K_{\infty}\right) \stackrel{\beta_{\xi}^{\vee}}{\longrightarrow} X\left(T_{f_{\xi}} / K_{\infty}\right)
$$

are pseudonull $O_{f_{\xi}}\left[\left[\Gamma_{K}\right]\right] \cong O_{f_{\xi}}\left[\left[T_{1}, T_{2}\right]\right]$ module for all but finitely many $\xi \in \mathfrak{X}(\mathcal{R})$. In particular, the equality

$$
C h_{O_{f_{\xi}}\left[\left[\Gamma_{K}\right]\right]}\left(\mathcal{X}\left(\mathcal{T}_{\mathcal{R}} / K_{\infty}\right) / P_{\xi} \mathcal{X}\left(\mathcal{T}_{\mathcal{R}} / K_{\infty}\right)\right)=C h_{O_{f_{\xi}}\left[\left[\Gamma_{K}\right]\right]}\left(X\left(T_{f_{\xi}} / K_{\infty}\right)\right)
$$

as ideals in $O_{f_{\xi}}\left[\left[\Gamma_{K}\right]\right]$ holds for all but finitely many arithmetic points.

Proof. The proof is different from the proof of Theorem 3.1 in two points. Here, we have to overcome the difficulty that there are possibly infinitely many primes in $K_{\infty}$ lying above a given prime in $K$. On the other hand, here we have the advantage that $\mathcal{R} \cong O[[W]]$, so that $P_{\xi}$ is principal.

Let us keep the notation as set up in the beginning of section 4 . For a finitely generated $O_{f_{\xi}}\left[\left[\Gamma_{K v}\right]\right]$ module $M$, recall $\operatorname{Ind}_{\Gamma_{K v}}^{\Gamma_{K}} M:=O_{f_{\xi}}\left[\left[\Gamma_{K}\right]\right] \otimes_{O_{f_{\xi}}}\left[\left[\Gamma_{K v}\right]\right] M$. Recall, by Shapiro's lemma $H_{i}\left(\Gamma_{K}, \operatorname{Ind}_{\Gamma_{K v}}^{\Gamma_{K}} M\right) \cong H_{i}\left(\Gamma_{K v}, M\right)$ for any $i \geq 0$.

Now, we have the commutative diagram with the natural maps

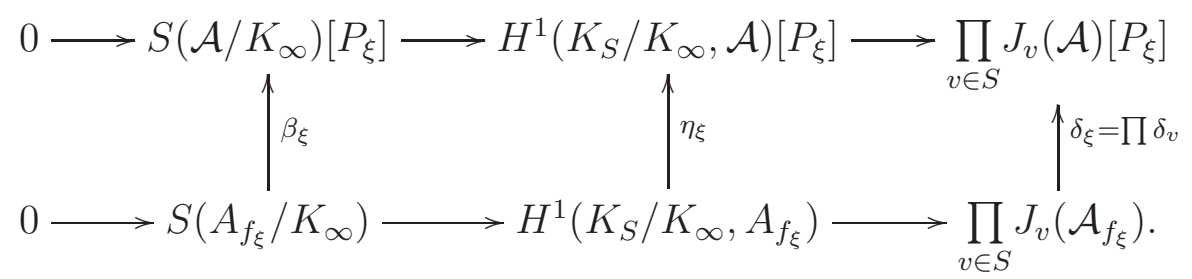

Here

$$
J_{v}(B):= \begin{cases}\left(\operatorname{Ind}_{\Gamma_{K v}}^{\Gamma_{K}} H^{1}\left(I_{v_{\infty}}, B\right)_{G_{v_{\infty}}}^{\vee}\right)^{\vee} & \text { if } v \in S, v \nmid p \\ \left(\operatorname{Ind}_{\Gamma_{K}}^{\Gamma_{K}} H^{1}\left(I_{v_{\infty}}, B^{-}\right)_{G_{v_{\infty}}}^{\vee}\right)^{\vee} & \text { if } v \mid p\end{cases}
$$

for $B=\mathcal{A}$ or $B=A_{f_{\xi}}$. Recall, once again $A_{f_{\xi}} \cong \mathcal{A}\left[P_{\xi}\right]$ and $A_{f_{\xi}}^{-} \cong \mathcal{A}^{-}\left[P_{\xi}\right]$. Also by our assumption that $\mathcal{R}$ is a power series ring, we have every $P_{\xi}$ is principal ideal in $\mathcal{R}$. Then $\eta_{\xi}$ is surjective with $\operatorname{ker}\left(\eta_{\xi}\right) \cong \mathcal{A}^{G_{K \infty}} / P_{\xi} A^{G_{K \infty}}$. To simplify notation, we put $\mathcal{T}:=\mathcal{T}_{\mathcal{R}}$ in the proof of this theorem. Then $\operatorname{ker}\left(\eta_{\xi}\right)^{\vee} \cong\left(\mathcal{T}_{G_{K_{\infty}}}^{\dagger}\right)_{\text {tor }}\left[P_{\xi}\right]$. Note, only finitely many $P_{\xi}$ divide the $\mathcal{R}$ characteristic ideal of finitely generated torsion $\mathcal{R}$ module $\left(\mathcal{T}_{G_{K_{\infty}}}^{\dagger}\right)_{\text {tor }}$. Hence we deduce that the $\operatorname{ker}\left(\beta_{\xi}\right)^{\vee}$ is finite (hence $O_{f_{\xi}}\left[\left[\Gamma_{K}\right]\right]$ pseudonull) for all but finitely many $\xi \in \mathfrak{X}(\mathcal{R})$.

As before, it suffices to show that $\operatorname{ker}\left(\delta_{\xi}\right)^{\vee}$ is $O_{f_{\xi}}\left[\left[\Gamma_{K}\right]\right]$ pseudonull leaving out finitely many exceptional $\xi$. Now it is easy to see that

$$
\left(\operatorname{ker}\left(\delta_{v}\right)\right)^{\vee}= \begin{cases}\operatorname{Ind}_{\Gamma_{K v}}^{\Gamma_{K}}\left(\left(\frac{\mathcal{A}^{I_{v_{\infty}}}}{P_{\xi} \mathcal{A}^{I_{v}}}\right)^{G_{v_{\infty}}}\right)^{\vee} \cong \operatorname{Ind}_{\Gamma_{K v}}^{\Gamma_{K}}\left(\mathcal{T}_{I_{v_{\infty}}}^{\dagger}\left[P_{\xi}\right]\right)_{G_{v_{\infty}}} & \text { if } v \in S, v \nmid p \\ \operatorname{Ind}_{\Gamma_{K v}}^{\Gamma_{K}}\left(\left(\frac{\mathcal{A}^{-I} v_{\infty}}{P_{\xi} \mathcal{A}^{-I_{v_{\infty}}}}\right)^{G_{v_{\infty}}}\right)^{\vee} \cong \operatorname{Ind}_{\Gamma_{K v}}^{\Gamma_{K}}\left(\mathcal{T}^{-\dagger}{ }_{I_{\infty}}\left[P_{\xi}\right]\right)_{G_{v_{\infty}}} & \text { if } v \mid p,\end{cases}
$$

But for a prime $v$ in $S$ not lying above $p$, we have $\mathcal{T}_{I_{v_{\infty}}}^{\dagger}\left[P_{\xi}\right] \cong\left(\mathcal{T}_{I_{v_{\infty}}}^{\dagger}\right)_{\text {tor }}\left[P_{\xi}\right]$ which is, as before, finite for all but finitely many $\xi$. Notice that $K_{\infty}$ contains $K_{\mathrm{cyc}}$ and hence the dimension of $\Gamma_{K v}$ as a $p$-adic Lie group is at least one. Hence Krull dimension 
of the commutative ring $O_{f_{\xi}}\left[\left[\Gamma_{K v}\right]\right]$ is at least 2. Thus $\left(\mathcal{T}_{I_{v_{\infty}}}^{\dagger}\left[P_{\xi}\right]\right)_{G_{K_{\infty}, v_{\infty}}}$ is finite and hence pseudonull as an $O_{f_{\xi}}\left[\left[\Gamma_{K v}\right]\right]$ module. Also, for a finitely generated $O_{f_{\xi}}\left[\left[\Gamma_{K v}\right]\right]$ module $M$ and for $i=0,1$ we have [Ve, Lemma 2.7(i)]

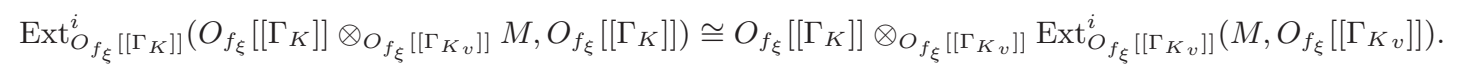

But a finitely generated $\Lambda$ ( for $\Lambda=O_{f_{\xi}}\left[\left[\Gamma_{K}\right]\right]$ or $\left.O_{f_{\xi}}\left[\left[\Gamma_{K v}\right]\right]\right)$ module $M$ is pseudonull if and only if $\operatorname{Ext}_{\Lambda}^{i}(M, \Lambda)=0$ for $i=0,1([\mathrm{Ve}])$. Thus we see that $\operatorname{Ind}_{\Gamma_{K v}}^{\Gamma_{K}}\left(\mathcal{T}_{I_{v_{\infty}}}^{\dagger}\left[P_{\xi}\right]\right)_{G_{v_{\infty}}}$ is pseudonull as a $O_{f_{\xi}}\left[\left[\Gamma_{K}\right]\right]$ module for all but finitely many $\xi$ and for any $v \in S, v \nmid p$. The same argument holds for a prime $v$ in $S$ dividing $p$ if we replace $\mathcal{T}$ by $\mathcal{T}^{-}$. Combining these for finitely many $v$ in $S$, we deduce that $\left(\operatorname{ker}\left(\delta_{\xi}\right)\right)^{\vee}$ is a pseudonull $O_{\xi}\left[\left[\Gamma_{K}\right]\right]$ module for all but finitely many $\xi \in \mathfrak{X}(\mathcal{R})$. This completes the proof.

Remark 4.7. We have analogous of remarks 3.2 and 3.3 for $K_{\infty}$ for the map $\mathcal{X}\left(\mathcal{T}_{\mathcal{R}}^{*} / K_{\infty}\right) / P_{\xi} \mathcal{X}\left(\mathcal{T}_{\mathcal{R}}^{*} / K_{\infty}\right) \stackrel{\beta_{\xi}^{*}}{\longrightarrow} X\left(T_{f_{\xi}} / K_{\infty}\right)$. Proceeding in Proposition 4.6 and using Corollary 4.3 we can get,

$$
C h_{O_{f_{\xi}}\left[\left[\Gamma_{K}\right]\right]}\left(\mathcal{X}\left(\mathcal{T}_{\mathcal{R}}^{*} / K_{\infty}\right) / P_{\xi} \mathcal{X}\left(\mathcal{T}_{\mathcal{R}}^{*} / K_{\infty}\right)\right)=C h_{O_{f_{\xi}}\left[\left[\Gamma_{K}\right]\right]}\left(X\left(T_{f_{\xi}}^{*} / K_{\infty}\right)\right)
$$

as ideals in $O_{f_{\xi}}\left[\left[\Gamma_{K}\right]\right]$ for all but finitely many $\xi \in \mathfrak{X}(\mathcal{R})$. Further, applying the involution $\iota$,

$$
C h_{f_{f_{\xi}}\left[\left[\Gamma_{K}\right]\right]}\left(\mathcal{X}\left(\mathcal{T}_{\mathcal{R}}^{*} / K_{\infty}\right)^{\iota} / P_{\xi} \mathcal{X}\left(\mathcal{T}_{\mathcal{R}}^{*} / K_{\infty}\right)^{\iota}\right)=C h_{O_{f_{\xi}}\left[\left[\Gamma_{K}\right]\right]}\left(X\left(T_{f_{\xi}}^{*} / K_{\infty}\right)^{\iota}\right)
$$

holds. Moreover, proceeding as in Corollary 3.5, we deduce that both $\mathcal{X}\left(\mathcal{T}_{\mathcal{R}} / K_{\infty}\right)$ and $\mathcal{X}\left(\mathcal{T}_{\mathcal{R}}^{*} / K_{\infty}\right)$ are finitely generated torsion modules over $\mathcal{R}\left[\left[\Gamma_{K}\right]\right]$.

Proposition 4.8. For any finitely generated $\mathcal{R}\left[\left[\Gamma_{K}\right]\right]$ module $U$, let $U^{0}$ denotes the maximal pseudonull $\mathcal{R}\left[\left[\Gamma_{K}\right]\right]$ submodule of $U$. Then $\frac{\mathcal{X}\left(\mathcal{T}_{\mathcal{R}} / K_{\infty}\right)^{0}}{P_{\xi} \mathcal{X}\left(\mathcal{T}_{\mathcal{R}} / K_{\infty}\right)^{0}}$ and $\frac{\mathcal{X}\left(\mathcal{T}_{\mathcal{R}}^{*} / K_{\infty}\right)^{0}}{P_{\xi} \mathcal{X}\left(\mathcal{T}_{\mathcal{R}}^{*} / K_{\infty}\right)^{0}}$ are pseudonull $O_{f_{\xi}}\left[\left[\Gamma_{K}\right]\right]$ modules for any $\xi \in \mathfrak{X}(\mathcal{R})$.

Proof. We proceed as in the proof of Proposition 3.7. Here the proof is different from Proposition 3.7 as the strict Selmer group and the usual Selmer group for $\mathcal{A}$ over $K_{\infty}$ may not coincide. As before, we will prove for $\mathcal{X}\left(\mathcal{T}_{\mathcal{R}} / K_{\infty}\right)$ only.

As in Proposition 3.7, it suffices to show $\mathcal{X}\left(\mathcal{T}_{\mathcal{R}} / F_{\infty}\right)\left[P_{\xi}\right]=0$ for every $\xi$. for $B=$ $\mathcal{A}$ or $A_{f_{\xi}}$, recall from section 1.2, the strict Selmer group $S^{\prime}\left(B / K_{\infty}\right) \hookrightarrow S\left(B / K_{\infty}\right)$. Then by corollary 4.3 and remark 4.7 , we deduce that all four groups $X\left(T_{f_{\xi}} / K_{\infty}\right)$, $X^{\prime}\left(T_{f_{\varepsilon}} / K_{\infty}\right), \mathcal{X}\left(\mathcal{T}_{\mathcal{R}} / K_{\infty}\right)$ and $\mathcal{X}^{\prime}\left(\mathcal{T}_{\mathcal{R}} / K_{\infty}\right)$ are finitely generated torsion modules over their respective Iwasawa algebras $O_{f_{\xi}}\left[\left[\Gamma_{K}\right]\right]$ and $\mathcal{R}\left[\left[\Gamma_{K}\right]\right]$. It follows that the maps defining the Greenberg Selmer groups and strict Selmer groups over $K_{\infty}$ are surjective ([Oc1, Theorem 4.10], [H-V, Theorem 7.12]). In other words, $\phi_{K_{\infty}}^{\prime B}$ is surjective for $B=\mathcal{A}$ or $B=A_{f_{\xi}}$ and we have the exact sequence

$$
\left.0 \longrightarrow S\left(B / K_{\infty}\right) \longrightarrow H^{1}\left(K_{S} / K_{\infty}, B\right) \longrightarrow \prod_{v \in S, v \nmid p} J_{v}\left(B / K_{\infty}\right) \prod_{v \mid p} J_{v}^{p}\left(B^{-} / K_{\infty}\right)\right) \longrightarrow 0
$$

Here $J_{v}\left(B / K_{\infty}\right)=\left(\operatorname{Ind}_{G_{v}}^{G} H^{1}\left(K_{\infty, v_{\infty}}, B\right)^{\vee}\right)^{\vee}$ and $J_{v}^{p}\left(B^{-} / K_{\infty}\right)=\left(\operatorname{Ind}_{G_{v}}^{G} H^{1}\left(I_{v_{\infty}}, B\right)^{G_{v_{\infty}}}\right)^{\vee}$. Hence we get another exact sequence

$$
0 \longrightarrow S^{\prime}\left(B / K_{\infty}\right) \longrightarrow \underset{29}{S\left(B / K_{\infty}\right) \longrightarrow U_{p}(B) \longrightarrow 0}
$$


with $U_{p}(B):=\left(\operatorname{Ind}_{\Gamma_{K v}}^{\Gamma_{K}} H^{1}\left(G_{v_{\infty}} / I_{v_{\infty}},\left(B^{-}\right)^{I_{v_{\infty}}}\right)^{\vee}\right)^{\vee}$. Therefore, it suffices to show that $\mathcal{X}^{\prime}\left(\mathcal{T}_{\mathcal{R}} / F_{\infty}\right)\left[P_{\xi}\right]=0$ and $U_{p}(\mathcal{A})^{\vee}\left[P_{\xi}\right]=0$. Now,

$$
H_{1}\left(G_{v_{\infty}} / I_{v_{\infty}}, \mathcal{A}^{-\vee}\right)\left[P_{\xi}\right]=H_{1}\left(G_{v_{\infty}} / I_{v_{\infty}}, \mathcal{T}_{\mathcal{R}}^{\dagger-}\right)\left[P_{\xi}\right]
$$

where $\mathcal{T}_{\mathcal{R}}^{\dagger}$ is a free $\mathcal{R}$ module of rank 1. As before, write $<\operatorname{Fr}_{v_{\infty}}>:=G_{v_{\infty}} / I_{v_{\infty}}$. Then the module in (51) is isomorphic to $\left(\mathcal{T}_{\mathcal{R}}^{\dagger}\left[\operatorname{Fr}_{v_{\infty}}\right]\right)\left[P_{\xi}\right]$. But $\left(\mathcal{T}_{\mathcal{R}}^{\dagger}\left[\operatorname{Fr}_{v_{\infty}}\right]\right)\left[P_{\xi}\right] \subset$ $\left(\mathcal{T}_{\mathcal{R}}^{\dagger}\right)\left[P_{\xi}\right]=0$ as $\mathcal{T}_{\mathcal{R}}^{\dagger-}$ is a free $\mathcal{R}$ module. Using this, it is plain from the definition of $U_{p}(\mathcal{A})$ that $U_{p}(\mathcal{A})^{\vee}\left[P_{\xi}\right]=0$.

We will show $\mathcal{X}^{\prime}\left(\mathcal{T}_{\mathcal{R}} / K_{\infty}\right)\left[P_{\xi}\right]=0$ to complete the proof of the proposition. Set

$$
\begin{aligned}
W_{\xi}^{\prime} & :=\prod_{v \in S, v \nmid p}\left(\operatorname{Ind}_{\Gamma_{K v}}^{\Gamma_{K}} H^{1}\left(K_{\infty, v_{\infty}}, A_{f_{\xi}}\right)^{\vee}\right)^{\vee} \prod_{v \mid p}\left(\operatorname{Ind}_{\Gamma_{K v}}^{\Gamma_{K}} H^{1}\left(K_{\infty, v_{\infty}}, A_{f_{\xi}}^{-}\right)^{\vee}\right)^{\vee} \text { and } \\
\mathcal{W}^{\prime} & :=\prod_{v \in S, v \nmid p}\left(\operatorname{Ind}_{\Gamma_{K v}}^{\Gamma_{K}} H^{1}\left(K_{\infty, v_{\infty}}, \mathcal{A}\right)^{\vee}\right)^{\vee} \prod_{v \mid p}\left(\operatorname{Ind}_{\Gamma_{K v}}^{\Gamma_{K}} H^{1}\left(K_{\infty, v_{\infty}}, \mathcal{A}^{-}\right)^{\vee}\right)^{\vee} \text { and }
\end{aligned}
$$

These are the 'local factors' used in the definition of $X^{\prime}\left(T_{f_{\xi}} / K_{\infty}\right)$ and $\mathcal{X}^{\prime}\left(\mathcal{T}_{\mathcal{R}} / K_{\infty}\right)$ respectively. Once again, as in proposition 3.7, this follows if we can show the map $W_{\xi}^{\prime} \longrightarrow \mathcal{W}^{\prime}\left[P_{\xi}\right]$ is surjective. Recall as $A\left[P_{\xi}\right] \cong A_{f_{\xi}}$. But for every prime $v_{\infty}$ of $K_{\infty}$ lying above a prime $S$ in $K$ not above $p$, the map $H^{1}\left(K_{\infty, v_{\infty}}, \mathcal{A}\left[P_{\xi}\right]\right) \longrightarrow$ $H^{1}\left(K_{\infty, v_{\infty}}, \mathcal{A}\right)\left[P_{\xi}\right]$ is surjective by Kummer theory. Similarly, for every $v_{\infty} \mid p$, $H^{1}\left(K_{\infty, v_{\infty}}, A_{f_{\xi}}^{-}\right) \longrightarrow H^{1}\left(K_{\infty, v_{\infty}}, \mathcal{A}^{-}\right)\left[P_{\xi}\right]$ is surjective. From these, it follows that $W_{\xi}^{\prime} \longrightarrow \mathcal{W}^{\prime}\left[P_{\xi}\right]$ is indeed surjective. This completes the proof of the proposition.

We now state our main theorem in the $\mathbb{Z}_{p}^{2}$ extension case.

Theorem 4.9. Let the notation be as before. Let $K$ be an imaginary quadratic field and $K \subset K_{\text {cyc }} \subset K_{\infty}$ be the unique $\mathbb{Z}_{p}^{\oplus 2}$ extension of $K$. Assume

(1) $\left(N_{\mathcal{R}}, D_{K}\right)=\left(p, D_{K}\right)=1$ i.e. $p$ splits in $K$ and the tame conductor $N_{\mathcal{R}}$ associated to the branch of the Hida family is coprime to the discriminant of $K$.

(2) (Irr): The residual representation $\bar{\rho}_{\mathcal{R}}$ is absolutely irreducible as $G_{\mathbb{Q}}$-module.

(3) (Dist) : The characters appearing on the diagonal of the local residual representation $\left.\bar{\rho}_{\mathcal{R}}\right|_{G_{p}}$ are distinct $(\bmod \mathfrak{m})$.

(4) $\mathcal{R}$ is a power series ring.

Then the functional equation holds for $\mathcal{X}\left(\mathcal{T}_{\mathcal{R}} / K_{\infty}\right)$ i.e. as an ideal in $\mathcal{R}\left[\left[\Gamma_{K}\right]\right]$,

$$
C h_{\mathcal{R}\left[\left[\Gamma_{K}\right]\right]}\left(\mathcal{X}\left(\mathcal{T}_{\mathcal{R}} / K_{\infty}\right)\right)=C h_{\mathcal{R}\left[\left[\Gamma_{K}\right]\right]}\left(\mathcal{X}\left(\mathcal{T}_{\mathcal{R}}^{*} / K_{\infty}\right)^{\iota}\right)
$$

Proof. By remark 4.7, we see that $\mathcal{X}\left(\mathcal{T}_{\mathcal{R}} / K_{\infty}\right)$ and $\mathcal{X}\left(\mathcal{T}_{\mathcal{R}}^{*} / K_{\infty}\right)$ are finitely generated torsion $\mathcal{R}\left[\left[\Gamma_{K}\right]\right]$ module. Let $S_{1}$ be the finite subset of $\mathfrak{X}(\mathcal{R})$ consisting of those $\xi$ for which $f_{\xi}$ is exceptional. Let $S_{2}$ be the finite subset of $\mathfrak{X}(\mathcal{R})$ for which the map $\beta_{\xi}$ in Proposition 4.6 is not a pseudo-isomorphism. Similarly $S_{3}$ be the finite subset of $\mathfrak{X}(\mathcal{R})$ for which the map $\beta_{\xi}^{*}$ in the remark 4.7 is not a pseudo-isomorphism. Let 
$\mathfrak{S}=S_{1} \cup S_{2} \cup S_{3}$ be the finite subset of $\mathfrak{X}(\mathcal{R})$. Then by Proposition 3.6, to prove the theorem, it suffices to show that for all $\xi \in \mathfrak{X}(\mathcal{R}) \backslash \mathfrak{S}$,

$$
C h_{O_{f_{\xi}}\left[\left[\Gamma_{K}\right]\right]}\left(\mathcal{X}\left(\mathcal{T}_{\mathcal{R}} / K_{\infty}\right) / P_{\xi} \mathcal{X}\left(\mathcal{T}_{\mathcal{R}} / K_{\infty}\right)\right)=C h_{O_{f_{\xi}}\left[\left[\Gamma_{K}\right]\right]}\left(\mathcal{X}\left(\mathcal{T}_{\mathcal{R}}^{*} / K_{\infty}\right)^{\iota} / P_{\xi} \mathcal{X}\left(\mathcal{T}_{\mathcal{R}} / K_{\infty}\right)^{\iota}\right)
$$

Using Proposition 4.6 and remark 4.7, (52) is in turn equivalent to showing for all $\xi \in \mathfrak{X}(\mathcal{R}) \backslash \mathfrak{S}$,

$$
C h_{f_{f_{\xi}}\left[\left[\Gamma_{K}\right]\right]}\left(X\left(\mathcal{T}_{f_{\xi}} / K_{\infty}\right)\right)=C h_{O_{f_{\xi}}\left[\left[\Gamma_{K}\right]\right]}\left(X\left(\mathcal{T}_{f_{\xi}}^{*} / K_{\infty}\right)^{\iota}\right)
$$

which follows from Theorem 4.5. This completes the proof of the proposition.

Remark 4.10. Our proof of Theorem 3.10 (respectively Theorem 4.9) covers the case when $\mathcal{X}\left(\mathcal{T}_{\mathcal{R}} / F_{\text {cyc }}\right)$ (respectively $\mathcal{X}\left(\mathcal{T}_{\mathcal{R}} / K_{\infty}\right)$ ) have non zero $\mathcal{R}[[\Gamma]]$ (respectively $\left.\mathcal{R}\left[\left[\Gamma_{K}\right]\right]\right)$ pseudonull submodules. We also allow the Selmer groups $X\left(T_{f_{\xi}} / F_{\text {cyc }}\right)$, $X\left(T_{f_{\xi}} / K_{\text {cyc }}\right), \xi \in \mathfrak{X}(\mathcal{R})$ to have positive $\mu$-invariant. Our proof of Theorem 4.9 works for both CM or non-CM Hida family.

Remark 4.11. Following Mazur's conjecture, the crucial assumption (Tor) for Hilbert modular forms in Theorem 3.10, is expected to be always true but has not been proven except in a few special cases. All the other conditions in Theorem 3.10 (respectively all the conditions in Theorem 4.9) are satisfied in many cases.

Remark 4.12. Keeping Leopoldt conjecture for a totally real field $F$ in mind, we do not pursue the $\mathbb{Z}_{p}^{2}$ extension case in case of Hilbert modular forms.

\section{REFERENCES}

[Bl] D. Blasius, Hilbert modular forms and the Ramanujan conjecture, Noncommutative geometry and number theory, Aspects Math., E37 (2006) 35-56.

[Ca] H. Carayol, Sur les représentations $l$-adiques associées aux formes modulaires de Hilbert, Ann. Sci. École Norm. Sup. (4), 19, no. 3, (1986) 409-468.

[C-S] J. Coates and R. Sujatha, Galois cohomology of elliptic curves, Tata Inst. Fund. Res. Stud. Math. 88 (2000).

[Di] M. Dimitrov, Automorphic symbols, $p$-adic $L$-functions and ordinary cohomology of Hilbert modular varieties. Amer. J. Math. 135 no. 4 (2013) 1117-1155.

[F-O] O. Fouquet and T. Ochiai, Control Theorems for Selmer groups of nearly ordinary deformations, J. Reine Angew. Math. 666 (2012), 163-187.

[Gr1] R. Greenberg, Iwasawa theory for $p$-adic representations, Algebraic number theory, Adv. Stud. Pure Math. 17 (1989) 97-137.

[Gr2] R. Greenberg, On the Structure of certain Galois cohomology group, Documenta Mathematica, Extra Volume Coates (2006), 335-391

[Hi1] H. Hida, Galois representations into $\mathrm{GL}_{2}\left(\mathbb{Z}_{p}[[X]]\right)$ attached to ordinary cusp forms, Invent. Math. 85 (1986), no. 3, 545-613.

[Hi2] H. Hida, On p-adic Hecke algebras for GL2 over totally real fields, Ann. of Math. (2) 128 no. 2, (1988) 295-384.

[H-M] Y. Hachimori and K. Matsuno, An analogue of Kida's formula for the Selmer groups of elliptic curves, J. Algebraic Geom. 8 (1999), no. 3, 581-601.

[H-V] Y. Hachimori and O. Venjakob, Completely faithful Selmer groups over Kummer extensions., Documenta Math., Kato Vol. (2003) 443-478.

[J-P] S. Jha and A. Pal, Algebraic functional equation for Hida family, International Journal of Number Theory, 10 (2014) No. 07, 1649-1674. 
[Ka] K. Kato, $p$-adic Hodge theory and values of zeta functions of modular forms, Cohomologies p-adiques et applications arithmétiques. III. Astérisque 295 (2004), ix, 117-290.

[M-W] B. Mazur and A. Wiles, On p-adic analytic families of Galois representations, Compositio Math. 59 (1986), 231-264.

[Ma] B. Mazur, Rational points of abelian varieties with values in towers of number fields, Invent. Math. 18, (1972) 183-266.

[M-SD] B. Mazur and P. Swinnerton-Dyer, Arithmetic of Weil Curves, Invent. Math 25 (1974) $1-61$.

[N-S-W] J. Neukirch, A. Schmidt and K. Wingberg, Cohomology of Number Fields, Grundlehren der Mathematischen Wissenschaften, Springer-Verlag (2000).

[Oc1] T. Ochiai, On the two-variable Iwasawa main Conjecture, Compositio. Math. 142 (2006), $1157-1200$.

[Oc2] T. Ochiai, Control Theorem for Greenberg's Selmer Groups of Galois Deformations, Journal of Number Theory $\mathbf{8 8}$ (2001), 59-85.

[Oc3] T. Ochiai, Euler system for Galois deformations, Ann Inst. Fourier Grenoble 55, 1 (2005) 113-146.

[Oc4] T. Ochiai, Several Variables $p$-adic $L$-Functions for Hida Families of Hilbert Modular Forms, Documenta Mathematica 17 (2012) 783-825.

[M-T-T] B. Mazur, J. Tate and J. Teitelbaum, On the $p$-adic analogues of the conjectures of Birch and Swinnerton-Dyer, Inventiones Math. 81 (1986) 1-48.

[Pe] B. Perrin-Riou, Groupes de Selmer et Accouplements; Cas Particulier des Courbes Elliptiques, Documenta Mathematica, Extra Volume Kato (2003) 725-760.

[Sa] D. B. Salazar, Cohomologie sur convergente des varitis modulaires de Hilbert et fonctions $L$ p-adiques, PhD Thesis, Universit Lille1 (2013).

[Su] R. Sujatha, Iwasawa theory and modular forms, Pure Appl. Math. Q. 2 (2006), no. 2, part 2, 519-538.

[S-U] C. Skinner and E. Urban, The Iwasawa main conjectures for $G L_{2}$, Inventiones Math. 195(1) (2014) 1-277.

[Ve] O. Venjakob, On the structure theory of the Iwasawa algebra of a $p$-adic Lie group, $J$. Eur. Math. Soc. 4 (2002), no. 3, 271-311.

[Wa] X. Wan, Iwasawa Main Conjecture for Hilbert Modular Forms, Preprint, (2013).

[Wi1] A. Wiles, On ordinary $\lambda$-adic representations associated to modular forms, Invent. Math. 94, no. 3, (1988) 529-573.

[Wi2] A. Wiles, The Iwasawa conjecture for totally real fields. Ann. of Math. (2) 131, no. 3, (1990) 493-540.

Somnath Jha, School of Physcial Sciences, Jawaharlal Nehru University, New DelHi - 110067, India

E-mail address: jhasomnath@gmail.com

Dipramit Majumdar, Indian Institute of Science Education and Research Pune, Pashan, Pune 411008, Maharashtra, India

E-mail address: dipramit@gmail.com 